\title{
FINITE ELEMENT APPROXIMATION OF ELLIPTIC HOMOGENIZATION PROBLEMS IN NONDIVERGENCE-FORM
}

\author{
Yves CapdeboscQ ${ }^{1}$, Timo Sprekeler ${ }^{2 *}$ And Endre Süli ${ }^{2}$
}

\begin{abstract}
We use uniform $W^{2, p}$ estimates to obtain corrector results for periodic homogenization problems of the form $A(x / \varepsilon): D^{2} u_{\varepsilon}=f$ subject to a homogeneous Dirichlet boundary condition. We propose and rigorously analyze a numerical scheme based on finite element approximations for such nondivergence-form homogenization problems. The second part of the paper focuses on the approximation of the corrector and numerical homogenization for the case of nonuniformly oscillating coefficients. Numerical experiments demonstrate the performance of the scheme.
\end{abstract}

Mathematics Subject Classification. 35B27, 35J15, 65N12, 65N30.

Received May 28, 2019. Accepted December 18, 2019.

\section{INTRODUCTION}

In this work we consider second-order elliptic equations of nondivergence structure, involving rapidly oscillating coefficients, of the form

$$
A\left(\frac{\cdot}{\varepsilon}\right): D^{2} u_{\varepsilon}:=\sum_{i, j=1}^{n} a_{i j}\left(\frac{\dot{c}}{\varepsilon}\right) \partial_{i j}^{2} u_{\varepsilon}=f \quad \text { in } \Omega,
$$

subject to the homogeneous Dirichlet boundary condition

$$
u_{\varepsilon}=0 \quad \text { on } \partial \Omega \text {. }
$$

Here we assume that $\Omega \subset \mathbb{R}^{n}$ is a sufficiently regular bounded domain, $\varepsilon>0$ is small, and that $A=\left(a_{i j}\right)$ : $\mathbb{R}^{n} \rightarrow \mathbb{R}^{n \times n}$ is a symmetric, uniformly elliptic and $(0,1)^{n}$-periodic matrix-valued function such that

$$
A \in W^{1, q}(Y) \text { for some } q>n,
$$

where $Y:=(0,1)^{n}$ denotes the unit cell; see Section 2.1. The main goal of this paper is to propose and analyze a numerical homogenization scheme for (1.1), (1.2) that is based on finite element approximations.

The theory of periodic homogenization is concerned with the limiting behavior of the solutions as the oscillation parameter $\varepsilon$ tends to zero. For the problem (1.1), (1.2) under consideration a classical homogenization

Keywords and phrases. Homogenization, nondivergence-form elliptic PDE, finite element methods.

1 Université de Paris, CNRS, Sorbonne Université, Laboratoire Jacques-Louis Lions UMR 7598, Paris, France.

2 University of Oxford, Mathematical Institute, Woodstock Road, Oxford OX2 6GG, UK.

*Corresponding author: timo.sprekeler@maths.ox.ac.uk

(C) The authors. Published by EDP Sciences, SMAI 2020

This is an Open Access article distributed under the terms of the Creative Commons Attribution License (https://creativecommons.org/licenses/by/4.0), which permits unrestricted use, distribution, and reproduction in any medium, provided the original work is properly cited. 
theorem (see [14], Sect. 3, Thm. 5.2) states that the solution sequence $\left(u_{\varepsilon}\right)_{\varepsilon>0}$ converges in an appropriate Sobolev space to the solution $u_{0}$ to the problem

$$
\left\{\begin{aligned}
A^{0}: D^{2} u_{0}=f & \text { in } \Omega, \\
u_{0}=0 & \text { on } \partial \Omega .
\end{aligned}\right.
$$

Here $A^{0} \in \mathbb{R}^{n \times n}$ is the constant matrix given by

$$
A^{0}=\int_{Y} A m
$$

and $m: \mathbb{R}^{n} \rightarrow \mathbb{R}$ is the invariant measure, i.e., the solution to the problem

$$
\left\{\begin{array}{l}
D^{2}:(A m)=0 \text { in } Y, \\
m \text { is } Y \text {-periodic, } \int_{Y} m=1
\end{array}\right.
$$

see Section 2 for further details. The task of numerical homogenization is the numerical approximation of the matrix $A^{0}$ and the solution $u_{0}$ to the homogenized problem (1.3). As it turns out, $u_{0}$ provides a good approximation to $u_{\varepsilon}$ in $H^{1}(\Omega)$, and by adding corrector terms it is possible to obtain an $H^{2}(\Omega)$-norm approximation. Note that the approximation of $(1.1),(1.2)$ by a standard $H^{2}(\Omega)$-conforming finite element method does not yield error bounds independent of $\varepsilon$, since for $s>0$ one has that

$$
\left\|u_{\varepsilon}\right\|_{H^{2+s}(\Omega)}=\mathcal{O}\left(\varepsilon^{-s}\right) .
$$

The motivation for investigating second-order elliptic problems in nondivergence-form comes from physics, engineering, as well as mathematical areas such as stochastic analysis. A notable example of a nonlinear PDE of nondivergence structure is the Hamilton-Jacobi-Bellman equation, which arises in stochastic control theory. The asymptotic behavior of PDEs with rapidly oscillating coefficients is also of importance when microinhomogeneous media are investigated.

Over the past decades significant work has been done on periodic homogenization of elliptic problems in divergence-form; numerical homogenization for nondivergence-form problems is however less developed.

The theory of homogenization of divergence-form problems such as

$$
\nabla \cdot\left(A\left(\frac{\cdot}{\varepsilon}\right) \nabla u_{\varepsilon}\right)+b\left(\frac{\dot{\varepsilon}}{\varepsilon}\right) \cdot \nabla u_{\varepsilon}=f \quad \text { in } \Omega
$$

with periodic and sufficiently regular $A: \mathbb{R}^{n} \rightarrow \mathbb{R}^{n \times n}$ and $b: \mathbb{R}^{n} \rightarrow \mathbb{R}^{n}$ is extensively covered in the books $[6,14,18,37]$. For divergence-form problems, various multiscale finite element methods (MsFEM) have been developed, which have the advantage over classical finite element methods of providing accurate approximations for very small values of $\varepsilon$ even for moderate values of the grid size. The book [19] by Efendiev and Hou contains a detailed overview of these methods.

It is important to note that although, if $A$ is sufficiently smooth, equation (1.1) can be rewritten in divergenceform,

$$
\nabla \cdot\left(A\left(\frac{\cdot}{\varepsilon}\right) \nabla u_{\varepsilon}\right)-\frac{1}{\varepsilon}(\operatorname{div} A)\left(\frac{\cdot}{\varepsilon}\right) \cdot \nabla u_{\varepsilon}=f \quad \text { in } \Omega,
$$

this equation does not fit into the framework of divergence-form homogenization problems such as (1.5), because of the $\varepsilon^{-1}$ term in front of the first-order term in (1.6). Such diffusion models with large drift have been considered by various authors $[7,8,17,20,30,31]$; they require either specific assumptions or the resolution of additional computationally onerous spectral problems.

For the theory of homogenization of nondivergence-form problems such as (1.1) we refer to the monograph [14] by Bensoussan et al., to the paper [10] by Avellaneda and Lin, and the references therein. In [13], Bensoussan 
et al. study the more general problem involving a Hamiltonian with quadratic growth. Numerical homogenization for nondivergence-form problems using finite difference schemes has been considered in [22] by Froese and Oberman.

The numerical method presented in this paper has resemblances with the finite element heterogeneous multiscale method (HMM). The HMM has been introduced in [38] by E and Engquist and has been successfully applied to many multiscale problems. For an overview of the field of finite element HMM, we refer to the articles [2-5] by Abdulle and co-authors, and the references therein. An a priori error analysis for the fully discrete finite element HMM for elliptic homogenization problems in divergence-form can be found in the work [1] by Abdulle. Concerning nondivergence-form problems, a finite difference HMM has recently been used for the numerical homogenization of second-order hyperbolic nondivergence-form problems by Arjmand and Kreiss [9].

The first step in the development of the proposed numerical homogenization scheme is the construction of a finite element method to obtain approximations $\left(m_{h}\right)_{h>0} \subset H_{\text {per }}^{1}(Y)$ to the invariant measure with optimal order convergence rate

$$
\left\|m-m_{h}\right\|_{L^{2}(Y)}+h\left\|m-m_{h}\right\|_{H^{1}(Y)} \lesssim h \inf _{\tilde{v}_{h} \in \tilde{M}_{h}}\left\|m-\left(\tilde{v}_{h}+1\right)\right\|_{H^{1}(Y)},
$$

where $\tilde{M}_{h}$ denotes the finite-dimensional subspace of $H_{\mathrm{per}}^{1}(Y)$ consisting of continuous $Y$-periodic piecewise linear functions on the triangulation with zero mean over $Y$; see Theorem 3.1.

Throughout this work, we use the notation $a \lesssim b$ for $a, b \in \mathbb{R}$ to denote that $a \leq C b$ for some constant $C>0$ that does not depend on $\varepsilon$ and the discretization parameters.

The second step is to obtain approximations $\left(A_{h}^{0}\right)_{h>0} \subset \mathbb{R}^{n \times n}$ to the constant matrix $A^{0}$; see Lemma 3.3. To this end, the integrand in (1.4) is replaced by its continuous piecewise linear interpolant and the invariant measure $m$ is replaced by the approximation $m_{h}$, i.e.,

$$
A_{h}^{0}:=\int_{Y} \mathcal{I}_{h}\left(A m_{h}\right)
$$

which can be computed exactly using an appropriate quadrature rule.

The third step is to perform an $H^{s}(\Omega)$-conforming $(s \in\{1,2\})$ finite element approximation for the problem

$$
\left\{\begin{aligned}
A_{h}^{0}: D^{2} u_{0}^{h}=f & \text { in } \Omega, \\
u_{0}^{h}=0 & \text { on } \partial \Omega,
\end{aligned}\right.
$$

on a family of triangulations of the computational domain $\bar{\Omega}$, parametrized by a discretization parameter $H>0$, measuring the granularity of the triangulation, to obtain $\left(u_{0}^{h, H}\right)_{h, H>0} \subset H^{s}(\Omega) \cap H_{0}^{1}(\Omega)$ with

$$
\left\|u_{0}^{h}-u_{0}^{h, H}\right\|_{H^{s}(\Omega)} \lesssim H\|f\|_{H^{s-1}(\Omega)}
$$

where the constant is independent of $h$; see Lemma 3.6. Note that for the sake of approximating $u_{0}$, an $H^{1}(\Omega)$ conforming finite element method is sufficient.

The approximation $\left(u_{0}^{h, H}\right)_{h, H>0} \subset H^{s}(\Omega) \cap H_{0}^{1}(\Omega)$ obtained by this procedure approximates $u_{0}$, i.e., the solution to (1.3), with convergence rate

$$
\left\|u_{0}-u_{0}^{h, H}\right\|_{H^{s}(\Omega)} \lesssim(h+H)\|f\|_{H^{s-1}(\Omega)}
$$

which can be improved to $\mathcal{O}\left(h^{2}+H\right)$ for more regular $A$; see Theorems 3.5, 3.9 and Remark 3.10.

Concerning the approximation of $u_{\varepsilon}$, i.e., the solution to (1.1), (1.2), we show in Section 2 that under certain assumptions on the domain and the right-hand side, one has that

$$
\left\|u_{\varepsilon}-u_{0}-\varepsilon^{2} \sum_{i, j=1}^{n} \chi_{i j}\left(\frac{\cdot}{\varepsilon}\right) \partial_{i j}^{2} u_{0}\right\|_{H^{2}(\Omega)} \lesssim \sqrt{\varepsilon}\left\|u_{0}\right\|_{W^{2, \infty}(\Omega)}+\varepsilon\left\|u_{0}\right\|_{H^{4}(\Omega)},
$$


where the corrector functions $\chi_{i j}: \mathbb{R}^{n} \rightarrow \mathbb{R}, i, j=1, \ldots, n$, are defined as the solutions to

$$
\left\{\begin{array}{l}
A: D^{2} \chi_{i j}=a_{i j}^{0}-a_{i j} \text { in } Y \\
\chi_{i j} \text { is } Y \text {-periodic, } \int_{Y} \chi_{i j}=0 .
\end{array}\right.
$$

This provides us with the estimate

$$
\left\|u_{\varepsilon}-u_{0}\right\|_{H^{1}(\Omega)}+\sum_{k, l=1}^{n}\left\|\partial_{k l}^{2} u_{\varepsilon}-\left(\partial_{k l}^{2} u_{0}+\sum_{i, j=1}^{n}\left(\partial_{k l}^{2} \chi_{i j}\right)(\cdot \dot{\varepsilon}) \partial_{i j}^{2} u_{0}\right)\right\|_{L^{2}(\Omega)}=\mathcal{O}(\sqrt{\varepsilon}),
$$

which shows that $u_{0}$ is a good $H^{1}(\Omega)$-norm approximation to $u_{\varepsilon}$ for small $\varepsilon$, and we show in Sections 3.2 and 3.3 how the above estimate can be used to obtain approximations to $D^{2} u_{\varepsilon}$. Note that in order to approximate $u_{\varepsilon}$ in the $H^{1}(\Omega)$-norm, it is sufficient to approximate $u_{0}$ in the $H^{1}(\Omega)$-norm. However, for an approximation of $D^{2} u_{\varepsilon}$ based on the above corrector estimate, we need to approximate $u_{0}$ in the $H^{2}(\Omega)$-norm.

In Section 3.4, we extend our results to the case of nonuniformly oscillating coefficients, i.e., to problems of the form

$$
\left\{\begin{aligned}
A\left(\cdot, \frac{\cdot}{\varepsilon}\right): D^{2} u_{\varepsilon}=f & \text { in } \Omega, \\
u_{\varepsilon}=0 & \text { on } \partial \Omega,
\end{aligned}\right.
$$

where $A=A(x, y): \Omega \times \mathbb{R}^{n} \rightarrow \mathbb{R}^{n \times n}$ is a symmetric, uniformly elliptic matrix-valued function that is $Y$-periodic in $y$ for fixed $x \in \Omega$, and such that

$$
A \in W^{2, \infty}\left(\Omega ; W^{1, q}(Y)\right) \quad \text { for some } \quad q>n .
$$

We prove the corrector estimate

$$
\left\|u_{\varepsilon}-u_{0}-\varepsilon^{2} \sum_{i, j=1}^{n} \chi_{i j}\left(\cdot, \frac{\cdot}{\varepsilon}\right) \partial_{i j}^{2} u_{0}\right\|_{H^{2}(\Omega)} \lesssim \sqrt{\varepsilon}\left\|u_{0}\right\|_{W^{2, \infty}(\Omega)}+\varepsilon\left\|u_{0}\right\|_{H^{4}(\Omega)},
$$

where $u_{0}$ is the solution to the homogenized problem corresponding to (1.7) and $\chi_{i j}$ are certain corrector functions. We then discuss the numerical approximation of $u_{\varepsilon}$ based on this corrector estimate; see Section 3.4.

In Section 4, we present numerical experiments for problems with periodic and nonuniformly oscillating coefficients, demonstrating the theoretical results.

Finally, in Section 5, we collect the proofs of the results contained in this work.

\section{HomogenizATION OF ELLIPTIC PROBLEMS IN NONDIVERGENCE-FORM}

In this section, we study the homogenization of elliptic problems in nondivergence-form with periodic coefficients. The outline of this section is as follows.

We provide the statement of the problem in Section 2.1, i.e., we define sets of assumptions for the domain, the coefficients and the right-hand side, ensuring well-posedness of the problem. In Section 2.2, we introduce the invariant measure and describe a well-known procedure for transforming the original nondivergence-form problem into a divergence-form problem. This is used in Section 2.3 in combination with uniform $W^{2, p}$ estimates to carry out the homogenization for the problem under consideration. Finally, we introduce correctors in Section 2.4 and derive $W^{2, p}$ homogenization results. 


\subsection{Framework}

We denote the unit cell in $\mathbb{R}^{n}$ by

$$
Y:=(0,1)^{n}
$$

and consider a symmetric matrix-valued function

$$
A=A^{\mathrm{T}}: \mathbb{R}^{n} \rightarrow \mathbb{R}^{n \times n}
$$

with the properties

$$
\left\{\begin{array}{l}
A \in W^{1, q}(Y) \text { for some } q \in(n, \infty] \\
A \text { is } Y \text {-periodic, } \\
\exists \lambda, \Lambda>0: \lambda|\xi|^{2} \leq A(y) \xi \cdot \xi \leq \Lambda|\xi|^{2} \quad \forall \xi, y \in \mathbb{R}^{n}
\end{array}\right.
$$

By Sobolev embedding, we then have that

$$
A \in C^{0, \alpha}\left(\mathbb{R}^{n}\right) \quad \text { for some } \quad 0<\alpha \leq 1 .
$$

For $\varepsilon>0$, we are concerned with the problem

$$
\left\{\begin{aligned}
A(\dot{-})): D^{2} u_{\varepsilon}=f & \text { in } \Omega \\
u_{\varepsilon}=0 & \text { on } \partial \Omega
\end{aligned}\right.
$$

where the triple $(\Omega, A, f)$ satisfies one of the following sets of assumptions.

Definition 2.1 (Sets of assumptions $\left.\mathcal{G}^{m, p}, \mathcal{H}^{m}\right)$. For $m \in \mathbb{N}_{0}$ and $p \in(1, \infty)$, we define the set of assumptions $\mathcal{G}^{m, p}$ as

$$
(\Omega, A, f) \in \mathcal{G}^{m, p} \Longleftrightarrow\left\{\begin{array}{r}
\Omega \subset \mathbb{R}^{n} \text { is a bounded } C^{2, \gamma} \text { domain, } \gamma \in(0,1), \\
A=A^{\mathrm{T}}: \mathbb{R}^{n} \rightarrow \mathbb{R}^{n \times n} \text { satisfies }(2.1), \\
f \in W^{m, p}(\Omega),
\end{array}\right.
$$

and the set of assumptions $\mathcal{H}^{m}$ as

$$
(\Omega, A, f) \in \mathcal{H}^{m} \Longleftrightarrow\left\{\begin{array}{r}
\Omega \subset \mathbb{R}^{n} \text { is a bounded convex domain, } \\
A=A^{\mathrm{T}}: \mathbb{R}^{n} \rightarrow \mathbb{R}^{n \times n} \text { satisfies }(2.1), \\
\exists \delta \in(0,1]: \frac{|A|^{2}}{(\operatorname{tr} A)^{2}} \leq \frac{1}{n-1+\delta} \text { in } \mathbb{R}^{n}, \\
f \in H^{m}(\Omega) .
\end{array}\right.
$$

Remark 2.2. For $n=2$, the Cordes condition, i.e., that there exists a $\delta \in(0,1]$ such that

$$
\frac{|A(y)|^{2}}{(\operatorname{tr} A(y))^{2}} \leq \frac{1}{n-1+\delta} \quad \forall y \in \mathbb{R}^{n}
$$

is a consequence of the uniform ellipticity condition. Indeed, for $A=A^{\mathrm{T}}: \mathbb{R}^{2} \rightarrow \mathbb{R}^{2 \times 2}$ satisfying (2.1), we have that

$$
\frac{|A(y)|^{2}}{(\operatorname{tr} A(y))^{2}}=1-\frac{2 \operatorname{det} A(y)}{(\operatorname{tr} A(y))^{2}} \leq 1-\frac{2 \lambda^{2}}{4 \Lambda^{2}}=\frac{1}{1+\delta} \quad \forall y \in \mathbb{R}^{n}
$$


with $\delta=\frac{\lambda^{2}}{2 \Lambda^{2}-\lambda^{2}} \in(0,1]$. Therefore, when $n=2$, the set $\mathcal{H}^{m}$ can be simplified to

$$
(\Omega, A, f) \in \mathcal{H}^{m} \Longleftrightarrow\left\{\begin{array}{r}
\Omega \subset \mathbb{R}^{n} \text { is a bounded convex domain, } \\
A=A^{\mathrm{T}}: \mathbb{R}^{n} \rightarrow \mathbb{R}^{n \times n} \text { satisfies }(2.1), \\
f \in H^{m}(\Omega) .
\end{array}\right.
$$

The following theorem asserts well-posedness of the problem (2.2); see Theorem 9.15 of [25] and Theorem 3 of [35].

Theorem 2.3 (Existence and uniqueness of strong solutions). Assume either that $(\Omega, A, f) \in \mathcal{G}^{0, p}$ for some $p \in(1, \infty)$, or that $(\Omega, A, f) \in \mathcal{H}^{0}$ and $p=2$. Then, for any $\varepsilon>0$, the problem (2.2) admits a unique solution $u_{\varepsilon} \in W^{2, p}(\Omega) \cap W_{0}^{1, p}(\Omega)$.

\subsection{Transformation into divergence-form}

We recall a well-known procedure to transform the problem (2.2) into divergence-form; see [10,14]. We use the notation

$$
W_{\mathrm{per}}(Y):=\left\{u \in H_{\mathrm{per}}^{1}(Y): \int_{Y} u=0\right\}
$$

Let us start by introducing the notion of invariant measure; see [14].

Lemma 2.4 (Invariant measure and solvability condition). Let $A=A^{\mathrm{T}}: \mathbb{R}^{n} \rightarrow \mathbb{R}^{n \times n}$ satisfy (2.1). Then, there exists a unique solution $m: \mathbb{R}^{n} \rightarrow \mathbb{R}$ to the problem

$$
\left\{\begin{array}{l}
D^{2}:(A m)=0 \text { in } Y \\
m \text { is } Y \text {-periodic, } \int_{Y} m=1 .
\end{array}\right.
$$

The function $m$ is called the invariant measure. We have that $m \in W^{1, q}(Y)$, see $[15,16]$, and there exist constants $\bar{m}, M>0$ such that

$$
0<\bar{m} \leq m(y) \leq M \quad \forall y \in \mathbb{R}^{n} .
$$

Moreover, for a $Y$-periodic function $g \in L^{2}\left(\mathbb{R}^{n}\right)$, the adjoint problem

$$
\left\{\begin{array}{l}
A: D^{2} u=g \text { in } Y \\
u \text { is } Y \text {-periodic, } \int_{Y} u=0,
\end{array}\right.
$$

admits a (unique) solution $u \in W_{\mathrm{per}}(Y)$ if and only if

$$
\langle g, m\rangle_{L^{2}(Y)}=0 .
$$

We note that the function $m$ is only in $W^{1, q}(Y)$ in general, and in particular it does not belong to $H^{2}(Y)$, as can be seen from the example chosen in Section 4.1. With the invariant measure $m$ at hand, we can easily convert the problem into divergence-form as follows. We define a matrix-valued function $B=\left(b_{i j}\right)_{1 \leq i, j \leq n}: \mathbb{R}^{n} \rightarrow \mathbb{R}^{n \times n}$ by

$$
b_{i j}:=\partial_{i} v_{j}-\partial_{j} v_{i}, \quad(1 \leq i, j \leq n),
$$

with $v_{l} \in W_{\text {per }}(Y)$ denoting the solution to

$$
\left\{\begin{array}{l}
-\Delta v_{l}=\operatorname{div}(A m) \cdot e_{l} \quad \text { in } Y \\
v_{l} \text { is } Y \text {-periodic, } \int_{Y} v_{l}=0
\end{array}\right.
$$


for $1 \leq l \leq n$. Since $A \in W^{1, q}(Y)$ and $m \in W^{1, q}(Y)$, by elliptic regularity one has that $v_{l} \in W^{2, q}(Y)$ for any $1 \leq l \leq n$. Hence, we have that

$$
B \in W^{1, q}(Y) .
$$

Further, we observe that $B$ is skew-symmetric, $Y$-periodic with zero mean over $Y$, and that

$$
\operatorname{div}(B)=-\operatorname{div}(A m) \quad \text { a.e. on } \mathbb{R}^{n} .
$$

Now we let

$$
A^{\mathrm{div}}:=A m+B \in W^{1, q}(Y)
$$

Then, since

$$
\operatorname{div}\left(A^{\operatorname{div}}\right)=0,
$$

and using the fact that $B$ is skew-symmetric, we obtain

$$
\nabla \cdot\left(A^{\operatorname{div}}\left(\frac{\cdot}{\varepsilon}\right) \nabla u_{\varepsilon}\right)=A^{\operatorname{div}}\left(\frac{\cdot}{\varepsilon}\right): D^{2} u_{\varepsilon}=(A m)\left(\frac{\cdot}{\varepsilon}\right): D^{2} u_{\varepsilon}
$$

that is, we have converted (2.2) into divergence-form:

$$
\left\{\begin{aligned}
\nabla \cdot\left(A^{\operatorname{div}}\left(\frac{\dot{-}}{\varepsilon}\right) \nabla u_{\varepsilon}\right) & =f m\left(\frac{\dot{-}}{\varepsilon}\right) & & \text { in } \Omega, \\
u_{\varepsilon} & =0 & & \text { on } \partial \Omega,
\end{aligned}\right.
$$

and it is straightforward to check that $A^{\text {div }}$ is $Y$-periodic, Hölder continuous on $\mathbb{R}^{n}$ and uniformly elliptic.

\subsection{Uniform $W^{2, p}$ estimates and homogenization theorem}

The transformation described in the previous section can be used to obtain uniform $W^{2, p}(\Omega)$ a priori estimates for the solution of (2.2), which are crucial in deriving homogenization results.

Theorem 2.5 (Uniform $W^{2, p}$ a priori estimates). Assume either that $(\Omega, A, f) \in \mathcal{G}^{0, p}$ for some $p \in(1, \infty)$, or that $(\Omega, A, f) \in \mathcal{H}^{0}$ and $p=2$. Then, for $\varepsilon \in(0,1]$, the solution $u_{\varepsilon} \in W^{2, p}(\Omega) \cap W_{0}^{1, p}(\Omega)$ to (2.2), whose existence and uniqueness are guaranteed by Theorem 2.3, satisfies

$$
\left\|u_{\varepsilon}\right\|_{W^{2, p}(\Omega)} \lesssim\|f\|_{L^{p}(\Omega)}
$$

with the constant absorbed into the notation $\lesssim$ being independent of $\varepsilon$.

This leads to a simple proof of the homogenization theorem for problem (2.2), using the compactness of the embedding $W^{2, p}(\Omega) \hookrightarrow W^{1, p}(\Omega)$ and the fact that we can rewrite the problem as (2.6).

Theorem 2.6 (Homogenization theorem for nondivergence-form problems). Assume either that $(\Omega, A, f) \in \mathcal{G}^{0, p}$ for some $p \in(1, \infty)$, or that $(\Omega, A, f) \in \mathcal{H}^{0}$ and $p=2$. Then the solution $u_{\varepsilon} \in W^{2, p}(\Omega) \cap W_{0}^{1, p}(\Omega)$ to $(2.2)$ converges weakly in $W^{2, p}(\Omega)$ to the solution $u_{0} \in W^{2, p}(\Omega) \cap W_{0}^{1, p}(\Omega)$ of the homogenized problem

$$
\left\{\begin{aligned}
A^{0}: D^{2} u_{0}=f & \text { in } \Omega, \\
u_{0}=0 & \text { on } \partial \Omega,
\end{aligned}\right.
$$

with $A^{0}=\left(a_{i j}^{0}\right)_{1 \leq i, j \leq n} \in \mathbb{R}^{n \times n}$ being the constant matrix whose entries are given by

$$
a_{i j}^{0}:=\int_{Y} a_{i j} m \quad(1 \leq i, j \leq n),
$$

where $m$ is the invariant measure, as defined in Lemma 2.4. 


\subsection{Correctors}

We show next that by adding corrector terms to the solution $u_{0}$ of the homogenized problem we obtain a $W^{2, p}$ convergence result.

Theorem 2.7 (Corrector estimate I). Assume either that $(\Omega, A, f) \in \mathcal{G}^{2, p}$ for some $p \in(1, \infty)$, or that $(\Omega, A, f) \in \mathcal{H}^{2}$ and $p=2$. Let $\varepsilon \in(0,1]$ and assume that

$$
u_{0} \in W^{4, p}(\Omega) .
$$

Introducing the corrector function $\chi_{i j}, 1 \leq i, j \leq n$, as the solution to

$$
\left\{\begin{array}{l}
A: D^{2} \chi_{i j}=a_{i j}^{0}-a_{i j} \text { in } Y, \\
\chi_{i j} \text { is } Y \text {-periodic, } \int_{Y} \chi_{i j}=0,
\end{array}\right.
$$

and a boundary corrector $\theta_{\varepsilon}$, as the solution to

$$
\left\{\begin{aligned}
A\left(\frac{\dot{-}}{\varepsilon}\right): D^{2} \theta_{\varepsilon} & =0 & & \text { in } \Omega, \\
\theta_{\varepsilon} & =-\sum_{i, j=1}^{n} \chi_{i j}\left(\frac{\cdot}{\varepsilon}\right) \partial_{i j}^{2} u_{0} & & \text { on } \partial \Omega,
\end{aligned}\right.
$$

the following bound holds:

$$
\left\|u_{\varepsilon}-u_{0}-\varepsilon^{2}\left(\sum_{i, j=1}^{n} \chi_{i j}\left(\frac{\cdot}{\varepsilon}\right) \partial_{i j}^{2} u_{0}+\theta_{\varepsilon}\right)\right\|_{W^{2, p}(\Omega)} \lesssim \varepsilon\left\|u_{0}\right\|_{W^{4, p}(\Omega)} .
$$

The following theorem shows that if $u_{0} \in W^{4, p}(\Omega) \cap W^{2, \infty}(\Omega)$, then we can absorb the term involving the boundary corrector into the right-hand side at the cost of powers of $\varepsilon$.

Theorem 2.8 (Corrector estimate II). Assume either that $(\Omega, A, f) \in \mathcal{G}^{2, p}$ for some $p \in(1, \infty)$, or that $(\Omega, A, f) \in \mathcal{H}^{2}$ and $p=2$. Let $\varepsilon \in(0,1]$ and assume that

$$
u_{0} \in W^{4, p}(\Omega) \cap W^{2, \infty}(\Omega) .
$$

Then,

$$
\left\|u_{\varepsilon}-u_{0}-\varepsilon^{2} \sum_{i, j=1}^{n} \chi_{i j}\left(\frac{\cdot}{\varepsilon}\right) \partial_{i j}^{2} u_{0}\right\|_{W^{2, p}(\Omega)} \lesssim \varepsilon^{\frac{1}{p}}\left\|u_{0}\right\|_{W^{2, \infty}(\Omega)}+\varepsilon\left\|u_{0}\right\|_{W^{4, p}(\Omega)} .
$$

Let us remark that $W^{4, p}(\Omega) \hookrightarrow W^{2, \infty}(\Omega)$ for $p>\frac{n}{2}$, i.e., assumption (2.10) is for $p>\frac{n}{2}$ a consequence of $u_{0} \in W^{4, p}(\Omega)$; in particular, for dimensions $n \in\{2,3\}$ and $p=2$, one can replace condition (2.10) by the sufficient condition $u_{0} \in H^{4}(\Omega)$.

Let us recall that $u_{0}$ is the solution to the elliptic constant-coefficient problem (2.7). For bounded convex polygonal domains $(n=2), u_{0} \in H^{4}(\Omega)$ can be ensured by assuming that $f \in H^{2}(\Omega)$ satisfies certain compatibility conditions at the corners of the domain. In the case of Poisson's equation on $\Omega=(0,1)^{2}$, a necessary and sufficient condition for $u_{0} \in H^{4}(\Omega) \cap H_{0}^{1}(\Omega)$ is that $f \in H^{2}(\Omega)$ and $f=0$ at the corners of $\Omega$; see [28]. We note that these conditions are satisfied for functions $f \in H^{2}(\Omega)$ such that $\operatorname{supp}(f) \Subset \Omega$; see [26]. 


\section{The NUMERICAL SCHEME}

In this section, we present and rigorously analyze the proposed numerical scheme. The outline of this section is as follows.

Section 3.1 is divided into three parts and discusses the numerical homogenization. In the first part, we approximate the invariant measure by a finite element method and provide a convergence result for the approximation. This is then used in the second part to obtain an approximation to the effective coefficients, i.e., to the constant matrix $A^{0}$. In the third part, we use a finite element method to discretize the homogenized problem and show convergence results for the approximation of the homogenized solution in $H^{1}(\Omega)$ and $H^{2}(\Omega)$, using the approximated effective coefficients, a comparison result, and two technical lemmata. Improvements to the convergence rates are given, provided more regularity on the coefficients is assumed.

In Section 3.2, we address the approximation of the corrector functions, presenting a method of successively approximating higher derivatives. We then use the homogenization results obtained in Section 2 and the approximations of the homogenized solution and the corrector functions from the previous subsections to approximate the original solution $u_{\varepsilon}$ in Section 3.3.

Finally, we study the case of nonuniformly oscillating coefficients in Section 3.4, derive homogenization results similar to the case of periodic coefficients and discuss the numerical homogenization for this case.

\subsection{Numerical homogenization scheme}

The first step is to approximate the invariant measure.

\subsubsection{Approximation of $m$}

For the approximation of the invariant measure $m$, we consider a shape-regular triangulation of $Y$ into triangles with longest edge $h>0$ and let

$$
\tilde{M}_{h} \subset W_{\text {per }}(Y)=\left\{v \in H_{\text {per }}^{1}(Y): \int_{Y} v=0\right\}
$$

be the finite-dimensional subspace of $W_{\text {per }}(Y)$ consisting of continuous $Y$-periodic piecewise linear functions on the triangulation with zero mean over $Y$. We assume that

$$
W_{\text {per }}(Y)=\overline{\bigcup_{h>0} \tilde{M}_{h}}
$$

Then we have the following approximation result for $m$.

Theorem 3.1 (Approximation of the invariant measure). Let $A=A^{\mathrm{T}}: \mathbb{R}^{n} \rightarrow \mathbb{R}^{n \times n}$ satisfy (2.1). Then, for $h>0$ sufficiently small, there exists a unique $\tilde{m}_{h} \in \tilde{M}_{h}$ such that

$$
\int_{Y}\left(A \nabla \tilde{m}_{h}+\tilde{m}_{h} \operatorname{div} A\right) \cdot \nabla \varphi_{h}=-\int_{Y}(\operatorname{div} A) \cdot \nabla \varphi_{h} \quad \forall \varphi_{h} \in \tilde{M}_{h}
$$

and writing

$$
m_{h}:=\tilde{m}_{h}+1,
$$

we have that

$$
\left\|m-m_{h}\right\|_{L^{2}(Y)}+h\left\|m-m_{h}\right\|_{H^{1}(Y)} \lesssim h \inf _{\tilde{v}_{h} \in \tilde{M}_{h}}\left\|m-\left(\tilde{v}_{h}+1\right)\right\|_{H^{1}(Y)},
$$

where $m$ is the invariant measure, as defined in Lemma 2.4. 
Remark 3.2. In particular, since

$$
\inf _{\tilde{v}_{h} \in \tilde{M}_{h}}\left\|m-\left(\tilde{v}_{h}+1\right)\right\|_{H^{1}(Y)}=o(1)
$$

we have that

$$
m_{h} \rightarrow m \quad \text { in } H^{1}(Y)
$$

as $h$ tends to zero.

\subsubsection{Approximation of $A^{0}$}

We use this finite element approximation of the invariant measure to obtain an approximation to the constant matrix

$$
A^{0}=\int_{Y} A m
$$

To this end, we first replace the invariant measure $m$ by the approximation $m_{h}$ from Theorem 3.1, and then replace the integrand by its piecewise linear interpolant,

$$
A_{h}^{0}:=\int_{Y} \mathcal{I}_{h}\left(A m_{h}\right)
$$

This integral can be computed exactly using an appropriate quadrature rule. The following lemma gives an error estimate for this approximation.

Lemma 3.3 (Approximation of $\left.A^{0}\right)$. Let $A=A^{\mathrm{T}}: \mathbb{R}^{n} \rightarrow \mathbb{R}^{n \times n}$ satisfy (2.1). Further, let $A^{0}=\left(a_{i j}^{0}\right) \in \mathbb{R}^{n \times n}$ be the constant matrix given by Theorem 2.6, let $m_{h}$ be the approximation to the invariant measure given by Theorem 3.1, and let $A_{h}^{0}=\left(a_{i j, h}^{0}\right) \in \mathbb{R}^{n \times n}$ be the matrix given by

$$
a_{i j, h}^{0}:=\int_{Y} \mathcal{I}_{h}\left(a_{i j} m_{h}\right), \quad 1 \leq i, j \leq n .
$$

Then, for $h>0$ sufficiently small, $A_{h}^{0}$ is elliptic and

$$
\max _{1 \leq i, j \leq n}\left|a_{i j}^{0}-a_{i j, h}^{0}\right| \lesssim h .
$$

\subsubsection{Approximation of $u_{0}$}

For the approximation of the solution $u_{0}$ to the homogenized problem, we use the following comparison result for the error committed when replacing $A^{0}$ by $A_{h}^{0}$.

Lemma 3.4 (Comparison result). Assume either that $(\Omega, A, f) \in \mathcal{G}^{0,2}$ or that $(\Omega, A, f) \in \mathcal{H}^{0}$. Let $A_{h}^{0} \in \mathbb{R}^{n \times n}$ be the approximation to $A^{0}$ as in Lemma 3.3. Then, for $h>0$ sufficiently small, we have that

$$
\left\|u_{0}-u_{0}^{h}\right\|_{H^{2}(\Omega)} \lesssim h\|f\|_{L^{2}(\Omega)},
$$

where $u_{0}^{h} \in H^{2}(\Omega) \cap H_{0}^{1}(\Omega)$ is the solution to the problem

$$
\left\{\begin{aligned}
A_{h}^{0}: D^{2} u_{0}^{h}=f & & \text { in } \Omega, \\
u_{0}^{h}=0 & & \text { on } \partial \Omega,
\end{aligned}\right.
$$

and $u_{0} \in H^{2}(\Omega) \cap H_{0}^{1}(\Omega)$ is the solution to the homogenized problem (2.7). 
Finally, we can use an $H_{0}^{1}(\Omega)$-conforming finite element approximation $u_{0}^{h, H}$ to the solution $u_{0}^{h}$ of $(3.2)$, satisfying the error bound

$$
\left\|u_{0}^{h}-u_{0}^{h, H}\right\|_{H^{1}(\Omega)} \lesssim H\left\|u_{0}^{h}\right\|_{H^{2}(\Omega)} \lesssim H\|f\|_{L^{2}(\Omega)}
$$

with constants independent of $h$. By the triangle inequality and the results obtained in this section, we have the following approximation result for $u_{0}$.

Theorem 3.5 ( $H^{1}$-norm approximation of $\left.u_{0}\right)$. Assume either that $(\Omega, A, f) \in \mathcal{G}^{0,2}$, or that $(\Omega, A, f) \in \mathcal{H}^{0}$. Then, the approximation $u_{0}^{h, H}$ obtained by the procedure described above satisfies

$$
\left\|u_{0}-u_{0}^{h, H}\right\|_{H^{1}(\Omega)} \lesssim(h+H)\|f\|_{L^{2}(\Omega)} .
$$

Let us now assume either that $(\Omega, A, f) \in \mathcal{G}^{1,2}$ or that $(\Omega, A, f) \in \mathcal{H}^{1}$. Further, assume that for $h>0$ sufficiently small, we have that $u_{0}^{h} \in H^{3}(\Omega)$ with

$$
\left\|u_{0}^{h}\right\|_{H^{3}(\Omega)} \lesssim\|f\|_{H^{1}(\Omega)}
$$

where the constant is independent of $h$. The following lemma provides two situations where this is satisfied.

Lemma 3.6. Let $(\Omega, A, f)$ be such that

(i) $(\Omega, A, f) \in \mathcal{G}^{1,2}$ with $\partial \Omega \in C^{3}$, or

(ii) $(\Omega, A, f) \in \mathcal{H}^{1}$ with $\Omega \subset \mathbb{R}^{2}$ being a polygon and $f \in H_{0}^{1}(\Omega)$.

Then, for $h>0$ sufficiently small, (3.3) holds.

In the proof of Lemma 3.6, we use the following result on the regularity of solutions to Poisson's problem on convex polygons; see also [26, 28, 29,32].

Lemma 3.7. Let $\Omega \subset \mathbb{R}^{2}$ be a convex polygonal domain and $f \in H_{0}^{1}(\Omega)$. Then the solution $u \in H_{0}^{1}(\Omega)$ to the problem

$$
\left\{\begin{aligned}
\Delta u=f & \text { in } \Omega \\
u=0 & \text { on } \partial \Omega
\end{aligned}\right.
$$

satisfies the bound

$$
\|u\|_{H^{3}(\Omega)} \lesssim\|f\|_{H^{1}(\Omega)} .
$$

Remark 3.8. The assumption $f \in H_{0}^{1}(\Omega)$ in Lemma 3.7 can be weakened provided $f$ satisfies certain compatibility conditions; see Theorem 5.1.2.4 from [26].

Then an $H^{2}(\Omega) \cap H_{0}^{1}(\Omega)$-conforming finite element approximation $u_{0}^{h, H}$ to the solution $u_{0}^{h}$ of (3.2), that satisfies the error bound

$$
\left\|u_{0}^{h}-u_{0}^{h, H}\right\|_{H^{2}(\Omega)} \lesssim H\left\|u_{0}^{h}\right\|_{H^{3}(\Omega)} \lesssim H\|f\|_{H^{1}(\Omega)},
$$

provides by Lemma 3.4 and the triangle inequality an approximation to $u_{0}$.

Theorem 3.9 $\left(H^{2}\right.$-norm approximation of $\left.u_{0}\right)$. Assume either that $(\Omega, A, f) \in \mathcal{G}^{1,2}$ or that $(\Omega, A, f) \in \mathcal{H}^{1}$, and assume (3.3). Then, the approximation $u_{0}^{h, H}$ obtained by the procedure described above satisfies

$$
\left\|u_{0}-u_{0}^{h, H}\right\|_{H^{2}(\Omega)} \lesssim(h+H)\|f\|_{H^{1}(\Omega)} .
$$


Remark 3.10 (Improvements). We note that if we assume that $A \in W^{2, \infty}(Y)$, then we have the following improved results.

(i) Approximation of $m$ : In this case, $m \in H^{2}(Y)$ and we have that

$$
\inf _{\tilde{v}_{h} \in \tilde{M}_{h}}\left\|m-\left(\tilde{v}_{h}+1\right)\right\|_{H^{1}(Y)} \leq\left\|m-\mathcal{I}_{h} m-\int_{Y}\left(m-\mathcal{I}_{h} m\right)\right\|_{H^{1}(Y)} \lesssim h\|m\|_{H^{2}(Y)},
$$

by choosing $\tilde{v}_{h}=\mathcal{I}_{h} m-\int_{Y} \mathcal{I}_{h} m$, and using an interpolation error bound. Therefore, Theorem 3.1 yields

$$
\left\|m-m_{h}\right\|_{L^{2}(Y)}+h\left\|m-m_{h}\right\|_{H^{1}(Y)} \lesssim h^{2}\|m\|_{H^{2}(Y)} .
$$

(ii) Approximation of $A^{0}$ : By an interpolation error bound and the fact that $m_{h}$ is piecewise linear, one has

$$
\left\|a_{i j} m_{h}-\mathcal{I}_{h}\left(a_{i j} m_{h}\right)\right\|_{L^{1}(Y)} \lesssim h^{2}\left\|a_{i j}\right\|_{W^{2, \infty}(Y)}\left\|m_{h}\right\|_{H^{1}(Y)} .
$$

Therefore, the proof of Lemma 3.3 yields

$$
\max _{1 \leq i, j \leq n}\left|a_{i j}^{0}-a_{i j, h}^{0}\right| \lesssim h^{2}\|A\|_{W^{2, \infty}(Y)}\|m\|_{H^{2}(Y)} \lesssim h^{2}\|A\|_{W^{2, \infty}(Y)} .
$$

(iii) Approximation of $u_{0}$ : It follows that the results of Lemma 3.4, Theorems 3.5 and 3.9 can be improved to second-order convergence in $h$, i.e.,

$$
\left\|u_{0}-u_{0}^{h, H}\right\|_{H^{s}(\Omega)} \lesssim\left(h^{2}\|A\|_{W^{2, \infty}(Y)}+H\right)\|f\|_{H^{s-1}(\Omega)}=\mathcal{O}\left(h^{2}+H\right),
$$

for $s=1,2$, respectively.

We note that second-order convergence with respect to $h$ could not have been obtained by using a piecewise constant approximation of $a_{i j} m_{h}$ instead of the piecewise linear approximation considered here. For the approximation of derivatives of $u_{0}$ of higher than second order, the post-processing method of Babuška [12] can be used to obtain error bounds in norms involving derivatives of higher order than the energy norm (the norm natural to the problem).

For bounded convex polygonal domains $\Omega \subset \mathbb{R}^{2}$, an $H^{2}$-conforming approximation to the solution of (3.2) can be obtained as follows. Assume that $f \in H_{0}^{1}(\Omega)$ so that (3.3) holds. Consider a shape-regular triangulation of $\Omega$ into triangles with longest edge $H>0$, and let

$$
V_{H} \subset H^{2}(\Omega) \cap H_{0}^{1}(\Omega)
$$

be an appropriate finite element space. In practice, the Hsieh-Clough-Tocher element and the Argyris element can be used as $H^{2}$-conforming elements. Then, for $h>0$ sufficiently small, standard finite element analysis can be used to show that there is a unique function $u_{0}^{h, H} \in V_{H}$ such that

$$
\int_{\Omega}\left(A_{h}^{0}: D^{2} u_{0}^{h, H}\right)\left(A_{h}^{0}: D^{2} \varphi_{H}\right)=\int_{\Omega} f\left(A_{h}^{0}: D^{2} \varphi_{H}\right) \quad \forall \varphi_{H} \in V_{H},
$$

and that the error bound (3.5) holds.

Further finite element approaches for approximating the solution of nondivergence-form problems include the conforming method [33] that makes use of a finite element Hessian, the discontinuous $h p$-Galerkin method $[35,36]$, the primal method [21] similar to an interior penalty discontinuous Galerkin method, the mixed finite element method [24], and the variational formulations presented in [23]. 


\subsection{Approximation of the corrector}

We now address problem (2.8) and present a method for $A \in W^{2, \infty}(Y)$. To simplify the notation and the arguments, we assume that we know the invariant measure $m$ and the matrix $A^{0}=\left(a_{i j}^{0}\right)_{1 \leq i, j \leq n}$ exactly instead of working with our approximation $A_{h}^{0}$.

For a given $Y$-periodic right-hand side $g \in W^{2, \infty}(Y)$, we consider the problem

$$
\left\{\begin{array}{l}
-\nabla \cdot(A \nabla \chi)+(\operatorname{div} A) \cdot \nabla \chi=-g \text { in } Y, \\
\chi \text { is } Y \text {-periodic, } \int_{Y} \chi=0 .
\end{array}\right.
$$

Obtaining an approximation for second-order derivatives via finite elements is not straightforward since the natural solution space is $W_{\text {per }}(Y)$. We present a method of successively approximating higher derivatives.

Let $\chi_{h}$ be a $W_{\text {per }}(Y)$-conforming finite element approximation to $\chi$, i.e.,

$$
\chi_{h} \in V_{h}, \quad \int_{Y} A \nabla \chi_{h} \cdot \nabla \varphi+\int_{Y} \varphi(\operatorname{div} A) \cdot \nabla \chi_{h}=-\int_{Y} g \varphi \quad \forall \varphi \in V_{h},
$$

with $V_{h} \subset W_{\text {per }}(Y)$ finite-dimensional, and satisfying the error estimate

$$
\left\|\chi_{h}-\chi\right\|_{H^{1}(Y)} \lesssim h .
$$

Let $r \in\{1, \ldots, n\}$ and write $\xi_{r}:=\partial_{r} \chi$. Then, using the equation

$$
-\nabla \cdot(A \nabla \chi)+(\operatorname{div} A) \cdot \nabla \chi=-g \text { in } Y,
$$

we find that in a weak sense, one has

$$
-\nabla \cdot\left(A \nabla \xi_{r}\right)+(\operatorname{div} A) \cdot \nabla \xi_{r}=-\partial_{r} g+\nabla \cdot\left(\partial_{r} A \nabla \chi\right)-\left(\operatorname{div}\left(\partial_{r} A\right)\right) \cdot \nabla \chi \quad \text { in } Y .
$$

Further, we claim that $\xi_{r} \in W_{\text {per }}(Y)$. Indeed, this follows from the regularity and periodicity of $\chi$ and

$$
\int_{Y} \partial_{r} \chi=\int_{\partial Y} \chi \nu \cdot e_{r}=0
$$

Therefore, $\xi_{r} \in W_{\text {per }}(Y)$ satisfies

$$
\left\{\begin{array}{l}
-\nabla \cdot\left(A \nabla \xi_{r}\right)+(\operatorname{div} A) \cdot \nabla \xi_{r}=-\partial_{r} g+\nabla \cdot\left(\partial_{r} A \nabla \chi\right)-\left(\operatorname{div}\left(\partial_{r} A\right)\right) \cdot \nabla \chi \quad \text { in } Y, \\
\xi_{r} \text { is } Y \text {-periodic, } \int_{Y} \xi_{r}=0 .
\end{array}\right.
$$

Now we use our $H^{1}$-conforming approximation for $\chi$ for the right-hand side and use a $W_{\text {per }}(Y)$-conforming finite element method for approximating the solution $v \in W_{\text {per }}(Y)$ to the following problem:

$$
\left\{\begin{array}{l}
-\nabla \cdot(A \nabla v)+(\operatorname{div} A) \cdot \nabla v=-\partial_{r} g+\nabla \cdot\left(\partial_{r} A \nabla \chi_{h}\right)-\left(\operatorname{div}\left(\partial_{r} A\right)\right) \cdot \nabla \chi_{h}-c \text { in } Y, \\
v \text { is } Y \text {-periodic, } \int_{Y} v=0
\end{array}\right.
$$

where $c$ is such that this problem admits a unique solution (such that the solvability condition (2.5) is satisfied). By looking at the problem for $v-\xi_{r}$, one obtains the comparison result

$$
\begin{aligned}
\left\|v-\xi_{r}\right\|_{H^{1}(Y)} & \lesssim\left\|\nabla \cdot\left(\partial_{r} A \nabla\left(\chi_{h}-\chi\right)\right)\right\|_{W_{\mathrm{per}}(Y)^{\prime}}+\left\|\left(\operatorname{div}\left(\partial_{r} A\right)\right) \cdot \nabla\left(\chi_{h}-\chi\right)\right\|_{W_{\mathrm{per}}(Y)^{\prime}} \\
& \lesssim\|A\|_{W^{2, \infty}(Y)}\left\|\chi_{h}-\chi\right\|_{H^{1}(Y)} \\
& \lesssim h\|A\|_{W^{2, \infty}(Y)}=\mathcal{O}(h) .
\end{aligned}
$$


Let $v_{h}$ be a $W_{\text {per }}(Y)$-conforming finite element approximation to the solution $v$ of (3.7) satisfying

$$
\left\|v_{h}-v\right\|_{H^{1}(Y)} \leq C h
$$

for some constant $C=C\left(\|A\|_{W^{2, \infty}(Y)}\right)>0$. Then, using the triangle inequality, we obtain

$$
\left\|v_{h}-\xi_{r}\right\|_{H^{1}(Y)} \leq C h
$$

for some constant $C=C\left(\|A\|_{W^{2, \infty}(Y)}\right)>0$. Using this procedure for $r=1, \ldots, n$, we eventually obtain approximations to derivatives of order up to two of $\chi$.

\subsection{Approximation of $\boldsymbol{u}_{\varepsilon}$}

We assume either that $(\Omega, A, f) \in \mathcal{G}^{2,2}$ or that $(\Omega, A, f) \in \mathcal{H}^{2}$. Let $n \in\{2,3\}, \varepsilon \in(0,1]$, and assume that

$$
u_{0} \in H^{4}(\Omega) \text {. }
$$

Then we know that (2.10) is satisfied, and by Theorem 2.8 we have that

$$
\left\|u_{\varepsilon}-u_{0}-\varepsilon^{2} \sum_{i, j=1}^{n} \chi_{i j}\left(\frac{\cdot}{\varepsilon}\right) \partial_{i j}^{2} u_{0}\right\|_{H^{2}(\Omega)} \lesssim \sqrt{\varepsilon}\left\|u_{0}\right\|_{W^{2, \infty}(\Omega)}+\varepsilon\left\|u_{0}\right\|_{H^{4}(\Omega)},
$$

where $u_{0}$ is the solution to the homogenized problem, and $\chi_{i j}$ are the corrector functions given as the solutions to (2.8). This result can be used to construct an approximation of $u_{\varepsilon}$, i.e., to the solution of problem (2.2) for small $\varepsilon$. We note that (3.8) implies that

$$
\left\|u_{\varepsilon}-u_{0}\right\|_{H^{1}(\Omega)}+\sum_{k, l=1}^{n}\left\|\partial_{k l}^{2} u_{\varepsilon}-\left(\partial_{k l}^{2} u_{0}+\sum_{i, j=1}^{n}\left(\partial_{k l}^{2} \chi_{i j}\right)(\dot{\bar{\varepsilon}}) \partial_{i j}^{2} u_{0}\right)\right\|_{L^{2}(\Omega)} \lesssim \sqrt{\varepsilon}\left\|u_{0}\right\|_{W^{2, \infty}(\Omega)}+\varepsilon\left\|u_{0}\right\|_{H^{4}(\Omega)}
$$

This leads to the following approximation result for $u_{\varepsilon}$.

Theorem 3.11 (Approximation of $u_{\varepsilon}$ ). In the situation described above, let $\left(u_{0, h}\right)_{h>0} \subset H^{2}(\Omega)$ be a family of $H^{2}$-conforming approximations for $u_{0}$ satisfying the error bound

$$
\left\|u_{0}-u_{0, h}\right\|_{H^{2}(\Omega)} \lesssim h\|f\|_{H^{1}(\Omega)},
$$

and for $1 \leq i, j, k, l \leq n$, let $\left(z_{i j, h}^{k l}\right)_{h>0} \subset L_{\mathrm{per}}^{2}(Y)$ be a family of $L^{2}$ approximations for $\partial_{k l}^{2} \chi_{i j}$ satisfying the error bound

$$
\left\|\partial_{k l}^{2} \chi_{i j}-z_{i j, h}^{k l}\right\|_{L^{2}(Y)} \lesssim h .
$$

Then, by writing

$$
u_{\varepsilon, h}^{k l}:=\partial_{k l}^{2} u_{0, h}+\sum_{i, j=1}^{n} z_{i j, h}^{k l}\left(\frac{\cdot}{\varepsilon}\right) \partial_{i j}^{2} u_{0, h},
$$

we have that

$$
\left\|u_{\varepsilon}-u_{0, h}\right\|_{H^{1}(\Omega)}+\sum_{k, l=1}^{n}\left\|\partial_{k l}^{2} u_{\varepsilon}-u_{\varepsilon, h}^{k l}\right\|_{L^{1}(\Omega)} \lesssim(\sqrt{\varepsilon}+h)\left\|u_{0}\right\|_{W^{2, \infty}(\Omega)}+\varepsilon\left\|u_{0}\right\|_{H^{4}(\Omega)}+h\|f\|_{H^{1}(\Omega)} .
$$


In connection with the previously described approximation of the homogenized solution $u_{0}$ and the corrector functions $\chi_{i j}$, note that Theorem 3.9 provides an $H^{2}(\Omega)$-conforming approximation to $u_{0}$ and the method presented in Section 3.2 provides $L_{\mathrm{per}}^{2}(Y)$ approximations for the second-order partial derivatives of $\chi_{i j}$, as required for the setting of Theorem 3.11 .

Let us conclude this section by remarking that if the second derivatives of the corrector functions are approximated in the space $L^{\infty}(Y)$ or if the solution to the homogenized problem is approximated in the space $W^{2, \infty}(\Omega)$, then one obtains by a similar proof an approximation result for the second derivatives of $u_{\varepsilon}$ in $L^{2}(\Omega)$.

Remark 3.12. If $\left(z_{i j, h}^{k l}\right)_{h>0} \subset L_{\text {per }}^{\infty}(Y)$ is a family of $L^{\infty}$ approximations for $\partial_{k l}^{2} \chi_{i j}$ satisfying the error bound

$$
\left\|\partial_{k l}^{2} \chi_{i j}-z_{i j, h}^{k l}\right\|_{L^{\infty}(Y)}=\mathcal{O}(h),
$$

and $\left(u_{0, h}\right)_{h>0}$ is as in Theorem 3.11, then we have that

$$
\left\|u_{\varepsilon}-u_{0, h}\right\|_{H^{1}(\Omega)}+\sum_{k, l=1}^{n}\left\|\partial_{k l}^{2} u_{\varepsilon}-u_{\varepsilon, h}^{k l}\right\|_{L^{2}(\Omega)}=\mathcal{O}(\sqrt{\varepsilon}+h) .
$$

The same holds true when $\left(u_{0, h}\right)_{h>0} \subset W^{2, \infty}(\Omega)$ is a family of $W^{2, \infty}$-conforming approximations for $u_{0}$ satisfying the error bound

$$
\left\|u_{0}-u_{0, h}\right\|_{W^{2, \infty}(\Omega)}=\mathcal{O}(h)
$$

and $\left(z_{i j, h}^{k l}\right)_{h>0}$ is as in Theorem 3.11 .

\subsection{Nonuniformly oscillating coefficients}

In this section, we discuss the case of nonuniformly oscillating coefficients, i.e., coefficients depending on $x$ and $\frac{x}{\varepsilon}$. We consider the problem

$$
\left\{\begin{aligned}
A\left(\cdot, \frac{\cdot}{\varepsilon}\right): D^{2} u_{\varepsilon}=f & \text { in } \Omega \\
u_{\varepsilon}=0 & \text { on } \partial \Omega
\end{aligned}\right.
$$

where the triple $(\Omega, A, f)$ satisfies one of the following sets of assumptions.

Definition 3.13 (Sets of assumptions $\mathcal{G}, \mathcal{H}$ ). We write

(i) $(\Omega, A, f) \in \mathcal{G}$ if and only if $\Omega \subset \mathbb{R}^{n}$ is a bounded $C^{2, \gamma}$ domain, $f \in L^{2}(\Omega)$, and $A=A^{\mathrm{T}}: \Omega \times \mathbb{R}^{n} \rightarrow \mathbb{R}^{n \times n}$ satisfies

$$
\left\{\begin{array}{l}
A=A(x, y) \in W^{2, \infty}\left(\Omega ; W^{1, q}(Y)\right) \text { for some } q \in(n, \infty] \\
A(x, \cdot) \text { is } Y \text {-periodic, } \\
\exists \lambda, \Lambda>0: \lambda|\xi|^{2} \leq A(x, y) \xi \cdot \xi \leq \Lambda|\xi|^{2} \quad \forall \xi, y \in \mathbb{R}^{n}, x \in \Omega
\end{array}\right.
$$

(ii) $(\Omega, A, f) \in \mathcal{H}$ if and only if $\Omega \subset \mathbb{R}^{n}$ is a bounded convex domain, $f \in L^{2}(\Omega)$, and $A=A^{\mathrm{T}}: \Omega \times \mathbb{R}^{n} \rightarrow \mathbb{R}^{n \times n}$ satisfies (3.11) and

$$
\exists \delta \in(0,1]: \frac{|A(x, y)|^{2}}{(\operatorname{tr} A(x, y))^{2}} \leq \frac{1}{n-1+\delta} \quad \forall(x, y) \in \Omega \times \mathbb{R}^{n}
$$

In view of Remark 2.2, we see that the Cordes condition (3.12) is always satisfied when $n=2$. Well-posedness of the problem (3.10) is guaranteed by the following theorem; see Theorem 9.15 of [25] and Theorem 3 of [35]. 
Theorem 3.14 (Existence and uniqueness of strong solutions). Assume either that $(\Omega, A, f) \in \mathcal{G}$, or that $(\Omega, A, f) \in \mathcal{H}$. Then, for any $\varepsilon>0$, the problem (3.10) admits a unique solution $u_{\varepsilon} \in H^{2}(\Omega) \cap H_{0}^{1}(\Omega)$.

As in Section 2, uniform a priori estimates for the solution to (3.10) allow passage to the limit in equation $(3.10)$; see $[13,14]$. The coefficient matrix of the homogenized problem now depends on the slow variable $x$, and is obtained by integrating against an invariant measure. Corrector results can then be shown as before.

Theorem 3.15 (Nonuniformly oscillating coefficients). Assume that $\varepsilon \in(0,1]$ and that either $(\Omega, A, f) \in \mathcal{G}$ or $(\Omega, A, f) \in \mathcal{H}$. Then, the following assertions hold:

(i) Uniform a priori estimate: The solution $u_{\varepsilon} \in H^{2}(\Omega) \cap H_{0}^{1}(\Omega)$ to (3.10) satisfies

$$
\left\|u_{\varepsilon}\right\|_{H^{2}(\Omega)} \lesssim\|f\|_{L^{2}(\Omega)} .
$$

(ii) Homogenization: The solution $u_{\varepsilon} \in H^{2}(\Omega) \cap H_{0}^{1}(\Omega)$ to (3.10) converges weakly in $H^{2}(\Omega)$ to the solution $u_{0} \in H^{2}(\Omega) \cap H_{0}^{1}(\Omega)$ of the homogenized problem

$$
\left\{\begin{aligned}
A^{0}: D^{2} u_{0}=f & \text { in } \Omega, \\
u_{0}=0 & \text { on } \partial \Omega,
\end{aligned}\right.
$$

with $A^{0}: \Omega \rightarrow \mathbb{R}^{n \times n}$ given by

$$
A^{0}(x):=\int_{Y} A(x, \cdot) m(x, \cdot)
$$

where $m=m(x, y)$ is the unique function $m: \Omega \times \mathbb{R}^{n} \rightarrow \mathbb{R}$ with $m \in C\left(\bar{\Omega} \times \mathbb{R}^{n}\right), 0<\bar{m} \leq m \leq M$ for some constants $\bar{m}, M>0$, such that

$$
\left\{\begin{array}{l}
D^{2}:(A(x, \cdot) m(x, \cdot))=0 \text { in } Y, \\
m(x, \cdot) \text { is } Y \text {-periodic, } \int_{Y} m(x, \cdot)=1,
\end{array}\right.
$$

for any fixed $x \in \Omega$. The function $m$ is called the invariant measure.

(iii) Corrector estimate: Assume that $f \in H^{2}(\Omega)$ and $u_{0} \in H^{4}(\Omega) \cap W^{2, \infty}(\Omega)$. Introducing the corrector function $\chi_{i j}, 1 \leq i, j \leq n$, as the solution to

$$
\left\{\begin{array}{l}
A(x, y): D_{y}^{2} \chi_{i j}(x, y)=a_{i j}^{0}(x)-a_{i j}(x, y), \quad(x, y) \in \Omega \times Y, \\
\chi_{i j}(x, \cdot) \text { is } Y \text {-periodic, } \int_{Y} \chi_{i j}(x, \cdot)=0
\end{array}\right.
$$

we have that

$$
\left\|u_{\varepsilon}-u_{0}-\varepsilon^{2} \sum_{i, j=1}^{n} \chi_{i j}\left(\cdot, \frac{\dot{\varepsilon}}{\varepsilon}\right) \partial_{i j}^{2} u_{0}\right\|_{H^{2}(\Omega)} \lesssim \sqrt{\varepsilon}\left\|u_{0}\right\|_{W^{2, \infty}(\Omega)}+\varepsilon\left\|u_{0}\right\|_{H^{4}(\Omega)} .
$$

Let us explain how the numerical scheme from Section 3.1 can be used for the numerical homogenization of (3.10).

First, we consider a shape-regular triangulation $\mathcal{T}_{H}$ on $\bar{\Omega}$ consisting of nodes $\left\{x_{i}\right\}_{i \in I}$ with grid size $H>0$, and a shape-regular triangulation $\mathcal{T}_{h}$ on $Y$ with grid size $h>0$. Then, for any $i \in I$, we can use the scheme from Section 3.1 (see Thm. 3.1) to obtain an approximation $m_{h}^{i} \in H^{1}(Y)$ to $m_{x_{i}}=m\left(x_{i}, \cdot\right)$ such that

$$
\left\|m_{x_{i}}-m_{h}^{i}\right\|_{L^{2}(Y)}+h\left\|m_{x_{i}}-m_{h}^{i}\right\|_{H^{1}(Y)} \lesssim h \inf _{\tilde{v}_{h} \in \tilde{M}_{h}}\left\|m_{x_{i}}-\left(\tilde{v}_{h}+1\right)\right\|_{H^{1}(Y)} .
$$


Further, we obtain that

$$
A_{h}^{0, i}:=\int_{Y} \mathcal{I}_{h}\left(A\left(x_{i}, \cdot\right) m_{h}^{i}\right)
$$

is an approximation to $A^{0}\left(x_{i}\right)$ (see Lem. 3.3),

$$
\left|A^{0}\left(x_{i}\right)-A_{h}^{0, i}\right| \lesssim h
$$

Now we define $A_{h, H}^{0}$ to be a continuous piecewise linear function on the triangulation $\mathcal{T}_{H}$ such that

$$
A_{h, H}^{0}\left(x_{i}\right)=A_{h}^{0, i}
$$

for all $i \in I$. Then, using (3.14) and denoting the continuous piecewise linear interpolant of a function $\phi$ on the triangulation $\mathcal{I}_{H}$ by $\mathcal{I}_{H} \phi$, we have

$$
\begin{aligned}
\left\|A^{0}-A_{h, H}^{0}\right\|_{L^{\infty}(\Omega)} & \leq\left\|A^{0}-\mathcal{I}_{H} A^{0}\right\|_{L^{\infty}(\Omega)}+\left\|\mathcal{I}_{H} A^{0}-A_{h, H}^{0}\right\|_{L^{\infty}(\Omega)} \\
& \lesssim\left\|A^{0}-\mathcal{I}_{H} A^{0}\right\|_{L^{\infty}(\Omega)}+h .
\end{aligned}
$$

We observe that, similarly to the proof of Lemma 3.4, we obtain that the solution $u_{0}^{h, H} \in H^{2}(\Omega) \cap H_{0}^{1}(\Omega)$ to

$$
\left\{\begin{aligned}
A_{h, H}^{0}: D^{2} u_{0}^{h, H}=f & & \text { in } \Omega, \\
u_{0}^{h, H}=0 & & \text { on } \partial \Omega,
\end{aligned}\right.
$$

satisfies, for $h, H>0$ sufficiently small,

$$
\left\|u_{0}-u_{0}^{h, H}\right\|_{H^{2}(\Omega)} \lesssim\left\|A^{0}-A_{h, H}^{0}\right\|_{L^{\infty}(\Omega)}\|f\|_{L^{2}(\Omega)}
$$

and in view of (3.15),

$$
\left\|u_{0}-u_{0}^{h, H}\right\|_{H^{2}(\Omega)} \lesssim\left(\left\|A^{0}-\mathcal{I}_{H} A^{0}\right\|_{L^{\infty}(\Omega)}+h\right)\|f\|_{L^{2}(\Omega)}=\mathcal{O}\left(H^{2}+h\right),
$$

where $u_{0}$ is the solution to the homogenized problem (3.13). Here we have used the interpolation estimate

$$
\left\|A^{0}-\mathcal{I}_{H} A^{0}\right\|_{L^{\infty}(\Omega)} \lesssim H^{2}\left\|A^{0}\right\|_{W^{2, \infty}(\Omega)},
$$

which follows from $A^{0} \in W^{2, \infty}(\Omega)$ and standard interpolation theory.

Remark 3.16. For problems in divergence-form, similar results have been derived previously using heterogeneous multiscale methods; see e.g., [1].

At this point, let us note that in contrast with our procedure of approximating the effective coefficient $A^{0}$ at the nodes of the coarse triangulation $\mathcal{T}_{H}$ and interpolating linearly, in the framework of the finite element heterogeneous multiscale method $A^{0}$ is typically approximated at the coarse integration nodes; see e.g., [1,2]. The use of piecewise linear interpolation allows us to obtain second-order convergence. Assuming more regularity on the coefficient $A(x, y)$ in $y$, as in Remark 3.10, the error in the approximation of the homogenized solution $u_{0}$ can be improved to order $\mathcal{O}\left(H^{2}+h^{2}\right)$. Finally, the solution to (3.16) can be approximated by a standard finite element method on the triangulation $\mathcal{T}_{H}$, which yields an approximation $u_{0, h, H} \in H^{2}(\Omega) \cap H_{0}^{1}(\Omega)$ to $u_{0}$ in the $H^{2}(\Omega)$-norm.

The approximation of $u_{\varepsilon}$ can be obtained based on the corrector estimate from Theorem 3.15 analogously as in Section 3.3. 


\section{NUMERICAL EXPERIMENTS}

In this section, we illustrate the theoretical results through numerical experiments. We provide an example for the case of periodic coefficients in Section 4.1, and one for the case of nonuniformly oscillating coefficients in Section 4.2. In both cases, we provide not only an example with an unknown $u_{0}$, but also a set-up with a known $u_{0}$ in order to test the approximation scheme for the homogenized solution.

The experiments demonstrate the performance of the scheme for the approximation of the invariant measure $m$, the effective coefficients $A^{0}$, the homogenized solution $u_{0}$, as well as the approximation of the solution $u_{\varepsilon}$ to the original problem for a fixed value of $\varepsilon$.

\subsection{Periodic coefficients}

We consider the homogenization problem

$$
\left\{\begin{aligned}
A\left(\frac{\cdot}{\varepsilon}\right): D^{2} u_{\varepsilon} & =f & & \text { in } \Omega, \\
u_{\varepsilon} & =0 & & \text { on } \partial \Omega,
\end{aligned}\right.
$$

on the domain

$$
\Omega:=Y=(0,1)^{2},
$$

with the matrix-valued map

$$
A: \mathbb{R}^{2} \rightarrow \mathbb{R}^{2 \times 2}, \quad A\left(y_{1}, y_{2}\right):=\left(\begin{array}{cc}
1+\arcsin \left(\sin ^{2}\left(\pi y_{1}\right)\right) & \sin \left(\pi y_{1}\right) \cos \left(\pi y_{1}\right) \\
\sin \left(\pi y_{1}\right) \cos \left(\pi y_{1}\right) & 2+\cos ^{2}\left(\pi y_{1}\right)
\end{array}\right)
$$

and the right-hand side $f: \Omega \rightarrow \mathbb{R}$ to be specified below. We observe that the matrix-valued function $A$ satisfies (2.1) with $q=\infty$. Further, note that

$$
A(y)=\left(a_{i j}\left(y_{1}\right)\right)_{1 \leq i, j \leq 2}
$$

depends only on the first coordinate of $y=\left(y_{1}, y_{2}\right) \in \mathbb{R}^{2}$; see Figure 1 .

In this case we know that the homogenized problem is given by

$$
\left\{\begin{aligned}
A^{0}: D^{2} u_{0}=f & \text { in } \Omega, \\
u_{0}=0 & \text { on } \partial \Omega,
\end{aligned}\right.
$$

where $A^{0} \in \mathbb{R}^{2 \times 2}$ denotes the constant matrix

$$
A^{0}=\int_{Y} A m
$$

with $m$ being the invariant measure

$$
m: \mathbb{R}^{2} \rightarrow \mathbb{R}, \quad m\left(y_{1}, y_{2}\right)=\left(\int_{0}^{1} \frac{\mathrm{d} t}{a_{11}(t)}\right)^{-1} \frac{1}{a_{11}\left(y_{1}\right)} ;
$$

see [22]. Explicit computation yields that

$$
\begin{array}{ll}
a_{11}^{0}=\left(\int_{0}^{1} \frac{\mathrm{d} t}{a_{11}(t)}\right)^{-1} & \approx 1.4684, \\
a_{12}^{0}=\left(\int_{0}^{1} \frac{\mathrm{d} t}{a_{11}(t)}\right)^{-1} \int_{0}^{1} \frac{a_{12}(t)}{a_{11}(t)} \mathrm{d} t=0, \\
a_{22}^{0}=\left(\int_{0}^{1} \frac{\mathrm{d} t}{a_{11}(t)}\right)^{-1} \int_{0}^{1} \frac{a_{22}(t)}{a_{11}(t)} \mathrm{d} t \approx 2.6037 .
\end{array}
$$




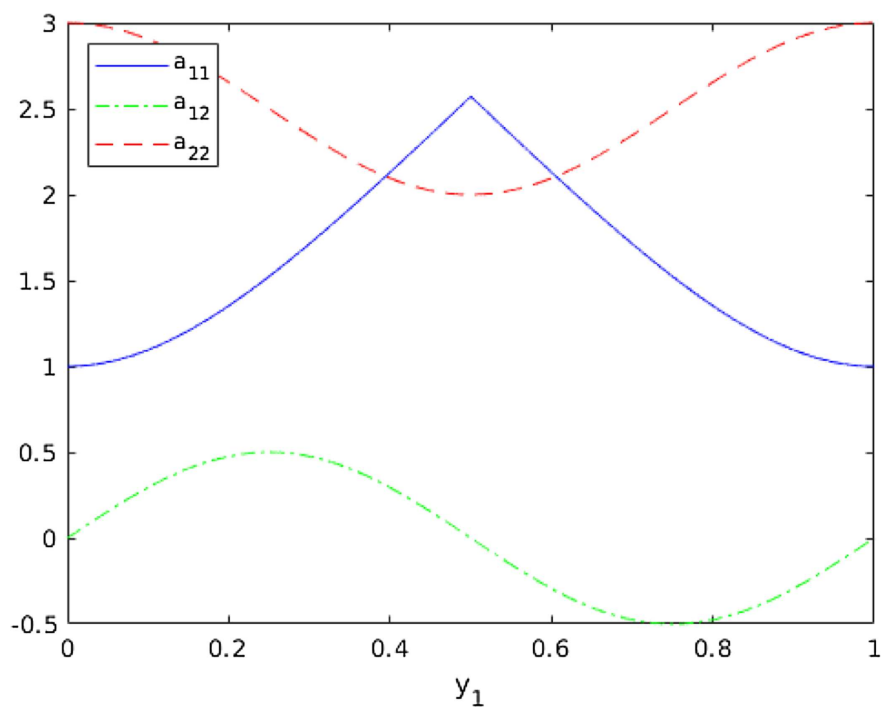

Figure 1. The functions $a_{i j}\left(y_{1}\right)$ plotted on the interval $(0,1)$.

We also note that for the corrector functions $\chi_{i j}(1 \leq i, j \leq 2)$, i.e., the solutions to

$$
\left\{\begin{array}{l}
A: D^{2} \chi_{i j}=a_{i j}^{0}-a_{i j} \text { in } Y, \\
\chi_{i j} \text { is } Y \text {-periodic, } \int_{Y} \chi_{i j}=0
\end{array}\right.
$$

we have that

$$
\partial_{k l}^{2} \chi_{i j}\left(y_{1}, y_{2}\right)=\left\{\begin{array}{cl}
\frac{a_{i j}^{0}-a_{i j}\left(y_{1}\right)}{a_{11}\left(y_{1}\right)} & \text { if } \quad k=l=1 \\
0 & \text { otherwise. }
\end{array}\right.
$$

Figure 2 shows the error in the approximation of $m$ and $A^{0}$ by the scheme presented in Section 3.1.

For the approximation of the invariant measure we observe convergence of order

$$
\left\|m-m_{h}\right\|_{L^{2}(Y)}=\mathcal{O}\left(h^{\frac{3}{2}}\right),
$$

and superconvergence of order $\mathcal{O}\left(h^{2}\right)$ for $h>0$ when grid points fall on the line $\left\{y_{1}=\frac{1}{2}\right\}$, which is the set along which $\partial_{1} m$ possesses a jump. The observed rate of convergence (4.3) is consistent with Theorem 3.1. Indeed, we have $m \in H^{\frac{3}{2}-\tilde{\varepsilon}}(Y)$ for any $\tilde{\varepsilon}>0$, and Theorem 3.1 yields

$$
\begin{aligned}
\left\|m-m_{h}\right\|_{L^{2}(Y)}+h\left\|m-m_{h}\right\|_{H^{1}(Y)} & \lesssim h \inf _{\tilde{v}_{h} \in \tilde{M}_{h}}\left\|m-\left(\tilde{v}_{h}+1\right)\right\|_{H^{1}(Y)} \\
& \lesssim h\left\|m-\mathcal{I}_{h} m-\int_{Y}\left(m-\mathcal{I}_{h} m\right)\right\|_{H^{1}(Y)} \\
& \lesssim h^{\frac{3}{2}-\tilde{\varepsilon}}\|m\|_{H^{\frac{3}{2}-\tilde{\varepsilon}}(Y)},
\end{aligned}
$$

by making the choice $\tilde{v}_{h}=\mathcal{I}_{h} m-\int_{Y} \mathcal{I}_{h} m$, and using an interpolation error bound. In connection with the superconvergence we note that $\left.m\right|_{\left(0, \frac{1}{2}\right) \times(0,1)} \in H^{2}\left(\left(0, \frac{1}{2}\right) \times(0,1)\right)$ and $\left.m\right|_{\left(\frac{1}{2}, 1\right) \times(0,1)} \in H^{2}\left(\left(\frac{1}{2}, 1\right) \times(0,1)\right)$. For the approximation of the matrix $A^{0}$, we observe second-order convergence. 

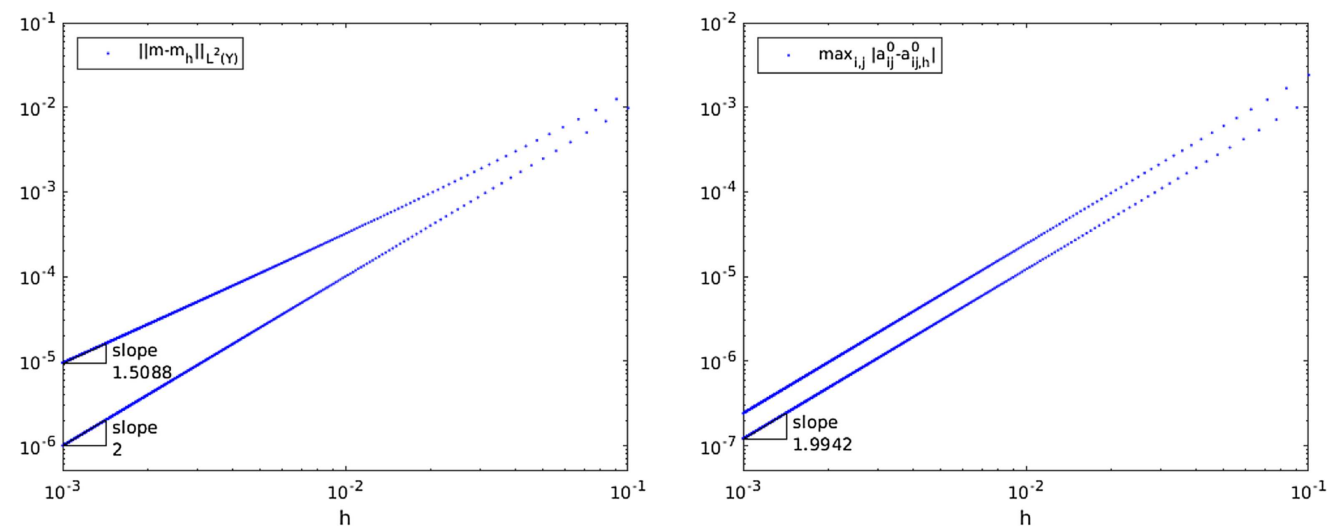

Figure 2. Approximation error for the invariant measure $m$ (left) and the matrix $A^{0}$ (right). Two curves are observed, corresponding to whether or not grid points fall on the line $\left\{y_{1}=\frac{1}{2}\right\}$, i.e., the set along which $\partial_{1} m$ exhibits a jump.

\subsubsection{Problem with a known $u_{0}$}

We consider the right-hand side given by

$$
f: \Omega \rightarrow \mathbb{R}, \quad f\left(x_{1}, x_{2}\right):=a_{22}^{0} x_{1}\left(x_{1}-1\right)+a_{11}^{0} x_{2}\left(x_{2}-1\right) .
$$

Then it is straightforward to check that the exact solution $u_{0} \in H^{2}(\Omega) \cap H_{0}^{1}(\Omega)$ to the homogenized problem (4.2) is given by

$$
u_{0}: \Omega \rightarrow \mathbb{R}, \quad u_{0}\left(x_{1}, x_{2}\right)=\frac{1}{2} x_{1}\left(x_{1}-1\right) x_{2}\left(x_{2}-1\right) .
$$

Note that we are in the situation $(\Omega, A, f) \in \mathcal{H}^{2}$, that $f=0$ at the corners of $\Omega$ and that $u_{0} \in H^{4}(\Omega)$.

We use the scheme presented in Section 3.1 to approximate the homogenized solution $u_{0}$, where we use the same mesh for approximating $m$ and $u_{0}$. The Hsieh-Clough-Tocher (HCT) element in FreeFem++ is used in the formulation (3.6) for the $H^{2}$ approximation of $u_{0}$; see [27]. The gradient on the boundary is set to be the gradient of an $H^{1}$ approximation by $\mathbb{P}_{2}$ elements on a fine mesh.

Concerning the approximation of $u_{\varepsilon}$, from Sections 2 and 3.3 we obtain that

$$
E_{\varepsilon}:=\left\|u_{\varepsilon}-u_{0}\right\|_{H^{1}(\Omega)}^{2}+\sum_{k, l=1}^{2}\left\|\partial_{k l}^{2} u_{\varepsilon}-\left(\partial_{k l}^{2} u_{0}+\sum_{i, j=1}^{2}\left(\partial_{k l}^{2} \chi_{i j}\right)\left(\frac{\cdot}{\varepsilon}\right) \partial_{i j}^{2} u_{0}\right)\right\|_{L^{2}(\Omega)}^{2}=\mathcal{O}(\varepsilon) .
$$

For the numerical approximation, we replace $u_{\varepsilon}$ by an $H^{2}$-conforming finite element approximation on a fine mesh, based on the formulation

$$
\text { Find } u_{\varepsilon} \in V: \quad \int_{\Omega} \frac{\operatorname{tr} A(\dot{\bar{\varepsilon}})}{|A(\dot{\bar{\varepsilon}})|^{2}} A\left(\frac{\dot{\varepsilon}}{\varepsilon}\right): D^{2} u_{\varepsilon} \Delta v=\int_{\Omega} \frac{\operatorname{tr} A(\dot{\bar{\varepsilon}})}{|A(\dot{\bar{\varepsilon}})|^{2}} f \Delta v \quad \forall v \in V,
$$

where $V:=H^{2}(\Omega) \cap H_{0}^{1}(\Omega)$. To this end, we use again the HCT element and set the gradient on the boundary to be the gradient of an $H^{1}$ approximation by $\mathbb{P}_{2}$ elements on a fine mesh.

Figure 3 shows the error in the approximation of $u_{0}$ and we observe second-order convergence. Further, with the exact $u_{0}$ being available, we can compute the error $E_{\varepsilon}$ for different values of $\varepsilon$; see Figure 3 . We observe first-order convergence as $\varepsilon$ tends to zero, as expected. 

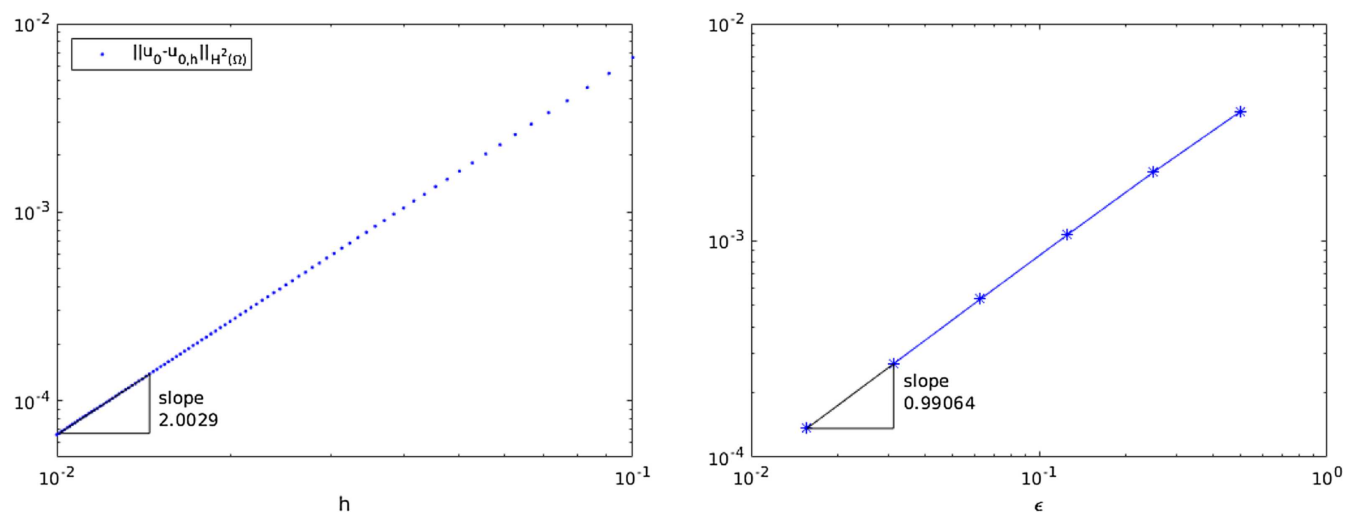

FiguRE 3. Approximation error for $u_{0}$ (left) and the error $E_{\varepsilon}$ in the approximation of $u_{\varepsilon}$ for different values of $\varepsilon$ (right).

\subsubsection{Problem with an unknown $u_{0}$}

Next, let us consider the problem (4.1) with the same domain $\Omega$ and matrix-valued function $A$ as before, but with the right-hand side given by

$$
f: \Omega \rightarrow \mathbb{R}, \quad f\left(x_{1}, x_{2}\right):=\exp \left(-\frac{1}{\frac{1}{2}-\left(x_{1}-\frac{1}{2}\right)^{2}-\left(x_{2}-\frac{1}{2}\right)^{2}}\right) .
$$

Note that now we are in the situation $(\Omega, A, f) \in \mathcal{H}^{2}$. Further, since the right-hand side $f \in H^{2}(\Omega)$ of the homogenized problem (4.2) satisfies $f=0$ at the corners of $\Omega$, the solution $u_{0}$ to (4.2) belongs to the class $H^{4}(\Omega)$; see Proposition 2.6 of [29].

As before, we use the scheme presented in Section 3.1 to approximate $m, A^{0}$ and $u_{0}$. Using the second-order $H^{2}(\Omega)$-conforming approximation $u_{0, h}$ to $u_{0}$ obtained as previously described,

$$
\left\|u_{0}-u_{0, h}\right\|_{H^{2}(\Omega)}=\mathcal{O}\left(h^{2}\right)
$$

we have that

$$
E_{\varepsilon}^{h}:=\left\|u_{\varepsilon}-u_{0, h}\right\|_{H^{1}(\Omega)}^{2}+\sum_{k, l=1}^{2}\left\|\partial_{k l}^{2} u_{\varepsilon}-\left(\partial_{k l}^{2} u_{0, h}+\sum_{i, j=1}^{2}\left(\partial_{k l}^{2} \chi_{i j}\right)\left(\frac{\cdot}{\varepsilon}\right) \partial_{i j}^{2} u_{0, h}\right)\right\|_{L^{2}(\Omega)}^{2}=\mathcal{O}\left(\varepsilon+h^{4}\right) .
$$

Figure 4 shows the error $E_{0.01}^{h}$ of the approximation of $u_{\varepsilon}$ for different grid sizes and $\varepsilon=\frac{1}{100}$ fixed. We observe fourth-order convergence in $h$ for the error as expected.

\subsection{Nonuniformly oscillating coefficients}

We consider the homogenization problem

$$
\left\{\begin{aligned}
A\left(\cdot, \frac{\cdot}{\varepsilon}\right): D^{2} u_{\varepsilon}=f & \text { in } \Omega \\
u_{\varepsilon}=0 & \text { on } \partial \Omega
\end{aligned}\right.
$$

on the domain

$$
\Omega:=Y=(0,1)^{2},
$$



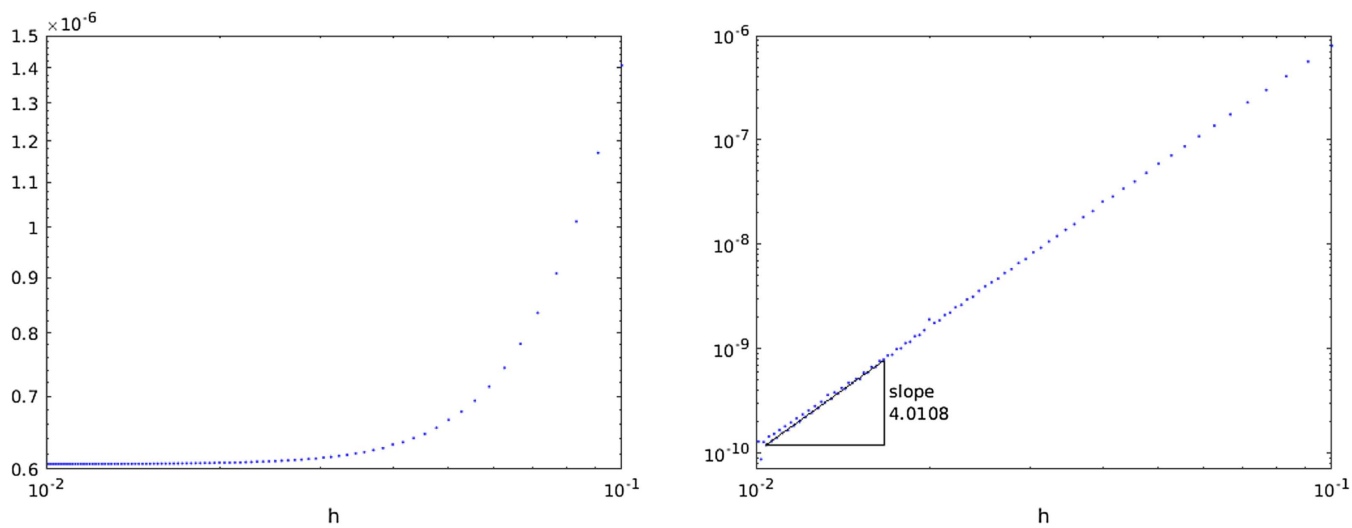

Figure 4 . The error $E_{0.01}^{h}$ in the approximation of $u_{\varepsilon}$ for a fixed value, $\varepsilon=\frac{1}{100}$, (left) and the error after subtraction of $6.0657 \times 10^{-7}$ (right), which is approximately the limit of $E_{0.01}^{h}$ in the figure on the left for this fixed value of $\varepsilon$ as $h$ tends to zero.

with the matrix-valued map $A: \Omega \times \mathbb{R}^{2} \rightarrow \mathbb{R}^{2 \times 2}$,

$$
(x, y)=\left(\left(x_{1}, x_{2}\right),\left(y_{1}, y_{2}\right)\right) \mapsto\left(\begin{array}{cc}
\mathrm{e}^{x_{1} x_{2}}+\frac{1}{4}|x|^{2} \arcsin \left(\sin ^{2}\left(\pi y_{1}\right)\right) & 0 \\
0 & 2+x_{2} \cos \left(2 \pi y_{2}+x_{1}\right)
\end{array}\right),
$$

and the right-hand side $f: \Omega \rightarrow \mathbb{R}$ to be specified below. We observe that the matrix-valued function $A$ satisfies (3.11) with $q=\infty$. Further, note that it is of the form

$$
A(x, y)=\operatorname{diag}\left(a_{11}\left(x, y_{1}\right), a_{22}\left(x, y_{2}\right)\right) .
$$

In this case we know that the homogenized problem is given by

$$
\left\{\begin{aligned}
A^{0}: D^{2} u_{0}=f & \text { in } \Omega, \\
u_{0}=0 & \text { on } \partial \Omega,
\end{aligned}\right.
$$

where $A^{0}: \Omega \rightarrow \mathbb{R}^{2 \times 2}$ is given by

$$
A^{0}(x)=\int_{Y} A(x, \cdot) m(x, \cdot)
$$

with $m$ being the invariant measure

$$
m: \Omega \times \mathbb{R}^{2} \rightarrow \mathbb{R}, \quad m(x, y)=\left(\int_{0}^{1} \int_{0}^{1} \frac{\mathrm{d} s \mathrm{~d} t}{a_{11}(x, s) a_{22}(x, t)}\right)^{-1} \frac{1}{a_{11}\left(x, y_{1}\right) a_{22}\left(x, y_{2}\right)}
$$

see [22]. Therefore, we have

$$
a_{i j}^{0}(x)=\delta_{i j}\left(\int_{0}^{1} \frac{\mathrm{d} t}{a_{i j}(x, t)}\right)^{-1}, \quad 1 \leq i, j \leq 2 .
$$

We also note that for the corrector functions $\chi_{i j}(1 \leq i, j \leq 2)$, i.e., the solutions to

$$
\left\{\begin{array}{l}
A(x, y): D_{y}^{2} \chi_{i j}(x, y)=a_{i j}^{0}(x)-a_{i j}(x, y), \quad(x, y) \in \Omega \times Y, \\
\chi_{i j}(x, \cdot) \text { is } Y \text {-periodic, } \int_{Y} \chi_{i j}(x, \cdot)=0
\end{array}\right.
$$


we have that

$$
\partial_{y_{k} y_{l}}^{2} \chi_{i j}(x, y)=\left\{\begin{array}{clc}
\frac{a_{11}^{0}(x)-a_{11}\left(x, y_{1}\right)}{a_{11}\left(x, y_{1}\right)} & \text { if } \quad i=j=k=l=1, \\
\frac{a_{22}^{0}(x)-a_{22}\left(x, y_{2}\right)}{a_{22}\left(x, y_{2}\right)} & \text { if } \quad i=j=k=l=2, \\
0 & \text { otherwise. }
\end{array}\right.
$$

\subsubsection{Problem with a known $u_{0}$}

We consider the right-hand side given by

$$
f: \Omega \rightarrow \mathbb{R}, \quad x=\left(x_{1}, x_{2}\right) \mapsto f(x):=a_{22}^{0}(x) x_{1}\left(x_{1}-1\right)+a_{11}^{0}(x) x_{2}\left(x_{2}-1\right) .
$$

Then it is straightforward to check that the exact solution $u_{0} \in H^{2}(\Omega) \cap H_{0}^{1}(\Omega)$ to the homogenized problem (4.5) is given by

$$
u_{0}: \Omega \rightarrow \mathbb{R}, \quad u_{0}\left(x_{1}, x_{2}\right)=\frac{1}{2} x_{1}\left(x_{1}-1\right) x_{2}\left(x_{2}-1\right) .
$$

Note that the assumptions of Theorem 3.15 (iii) are satisfied.

For $H>0$ such that $\frac{1}{H} \in \mathbb{N}$, we take a triangulation $\mathcal{T}_{H}$ on $\bar{\Omega}$ consisting of nodes $\{(s H, r H)\}_{s, r=0, \ldots, 1 / H}$, and a triangulation $\mathcal{T}_{h}$ on $Y$ with grid size $h=\frac{H}{4}$. We use the scheme presented in Section 3.4 to approximate $A^{0}$ and $u_{0}$, and we observe second-order convergence; see Figure 5.

For the approximation of $u_{\varepsilon}$, Theorem 3.15 yields

$$
E_{\varepsilon}:=\left\|u_{\varepsilon}-u_{0}\right\|_{H^{1}(\Omega)}^{2}+\sum_{k, l=1}^{2}\left\|\partial_{k l}^{2} u_{\varepsilon}-\left(\partial_{k l}^{2} u_{0}+\sum_{i, j=1}^{2}\left(\partial_{y_{k} y_{l}}^{2} \chi_{i j}\right)\left(\cdot, \frac{\cdot}{\varepsilon}\right) \partial_{i j}^{2} u_{0}\right)\right\|_{L^{2}(\Omega)}^{2}=\mathcal{O}(\varepsilon) .
$$

For the numerical approximation, we replace $u_{\varepsilon}$ by an $H^{2}$-conforming finite element method on a fine mesh, based on the formulation

$$
\text { Find } u_{\varepsilon} \in V: \quad \int_{\Omega} \frac{\operatorname{tr} A(\cdot, \dot{\bar{\varepsilon}})}{\mid A\left(\cdot,\left.\frac{\dot{\bar{\varepsilon}}}{\varepsilon}\right|^{2}\right.} A\left(\cdot, \frac{\dot{\varepsilon}}{\varepsilon}\right): D^{2} u_{\varepsilon} \Delta v=\int_{\Omega} \frac{\operatorname{tr} A(\cdot, \dot{\bar{\varepsilon}})}{|A(\cdot, \dot{\bar{\varepsilon}})|^{2}} f \Delta v \quad \forall v \in V,
$$

where $V:=H^{2}(\Omega) \cap H_{0}^{1}(\Omega)$. To this end, we use again the HCT element and set the gradient on the boundary to be the gradient of an $H^{1}$-conforming approximation by $\mathbb{P}_{2}$ elements on a fine mesh.

\subsubsection{Problem with an unknown $u_{0}$}

Finally, let us consider the problem (4.4) with the same domain $\Omega$ and matrix-valued function $A$ as before, but with the right-hand side given by

$$
f: \Omega \rightarrow \mathbb{R}, \quad f\left(x_{1}, x_{2}\right):=\exp \left(-\frac{1}{\frac{1}{2}-\left(x_{1}-\frac{1}{2}\right)^{2}-\left(x_{2}-\frac{1}{2}\right)^{2}}\right) .
$$

Note that we are in the situation $(\Omega, A, f) \in \mathcal{H}$. Using the second-order $H^{2}$-conforming approximation $u_{0, H}$ to $u_{0}$ obtained as previously described (again with $h=\frac{H}{4}$ ),

$$
\left\|u_{0}-u_{0, H}\right\|_{H^{2}(\Omega)}=\mathcal{O}\left(H^{2}\right)
$$

we have that

$$
E_{\varepsilon}^{H}:=\left\|u_{\varepsilon}-u_{0, H}\right\|_{H^{1}(\Omega)}^{2}+\sum_{k, l=1}^{2}\left\|\partial_{k l}^{2} u_{\varepsilon}-\left(\partial_{k l}^{2} u_{0, H}+\sum_{i, j=1}^{2}\left(\partial_{y_{k} y_{l}}^{2} \chi_{i j}\right)\left(\cdot, \frac{\dot{ }}{\varepsilon}\right) \partial_{i j}^{2} u_{0, H}\right)\right\|_{L^{2}(\Omega)}^{2}=\mathcal{O}\left(\varepsilon+H^{4}\right) .
$$

Figure 6 shows the error $E_{0.02}^{H}$ of the approximation of $u_{\varepsilon}$ for different grid sizes and $\varepsilon=\frac{1}{50}$ fixed. We observe fourth-order convergence in $H$ for the error as expected. 

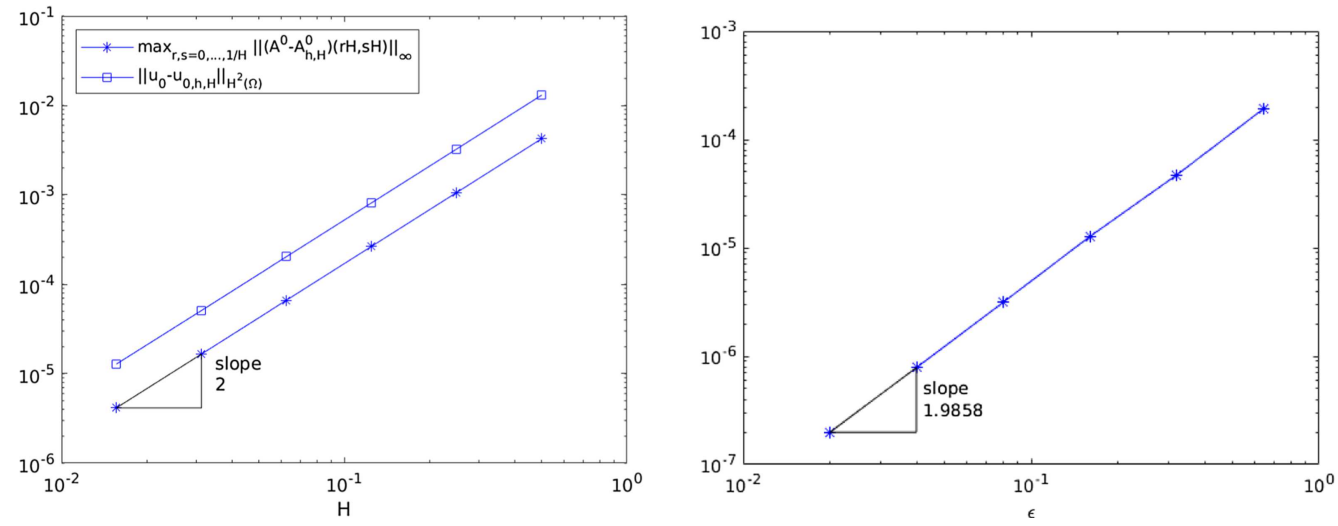

FiguRE 5. Approximation error for $A^{0}$ and $u_{0}$ for different values of $H$, using $h=\frac{H}{4}$, (left) and the error $E_{\varepsilon}$ in the approximation of $u_{\varepsilon}$ for different values of $\varepsilon(r i g h t)$.
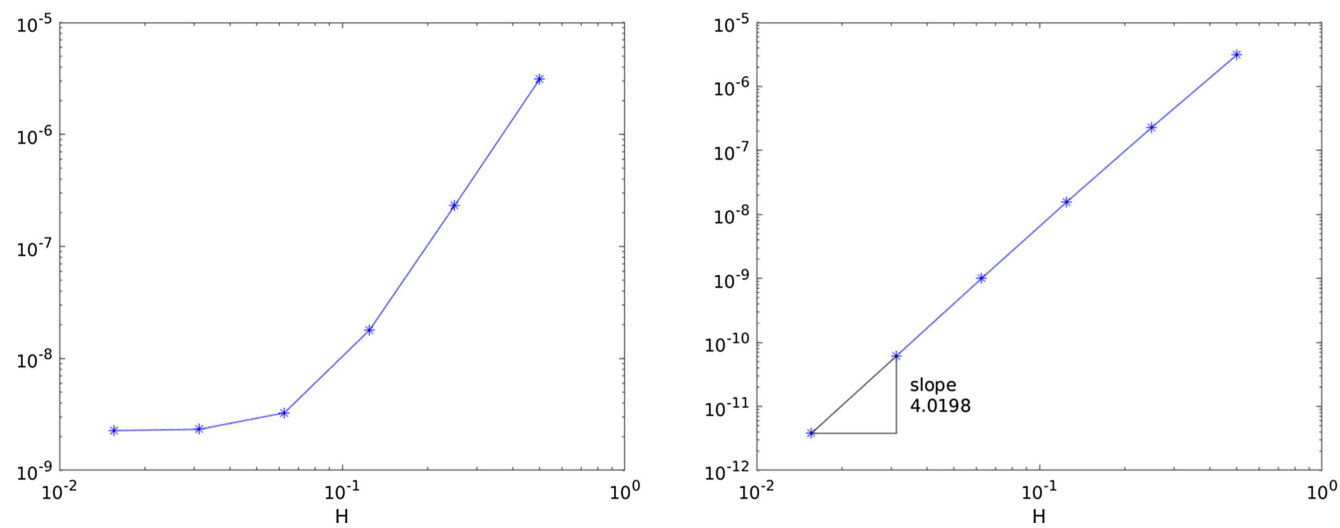

Figure 6 . The error $E_{0.02}^{H}$ in the approximation of $u_{\varepsilon}$ for a fixed value, $\varepsilon=\frac{1}{50}$, (left) and the error after subtraction of $2.2653 \times 10^{-9}$ (right), which is approximately the limit of $E_{0.02}^{H}$ in the figure on the left for this fixed value of $\varepsilon$ as $H$ tends to zero.

\section{Collection of Proofs}

In this section, we provide the proofs to the results presented in this paper. This section is divided into a part containing the proofs of the homogenization results from Section 2 as well as the proof of Theorem 3.15, and a second part containing the proofs of numerical results from Section 3, except for the technical Lemmata 3.6 and 3.7, which can be found in the last part of this section.

\subsection{Proofs of the homogenization results}

Proof of Theorem 2.5. Let us first assume that $(\Omega, A, f) \in \mathcal{G}^{0, p}$ for some $p \in(1, \infty)$. We showed in the previous section that we can transform problem (2.2) into the divergence-form problem (2.6), where $A^{\text {div }}: \mathbb{R}^{n} \rightarrow \mathbb{R}^{n \times n}$ is a $Y$-periodic, Hölder continuous, and uniformly elliptic matrix-valued function satisfying

$$
\operatorname{div}\left(A^{\operatorname{div}}\right)=0 .
$$


Therefore, we can apply Theorem D from [11] to problem (2.6) to obtain

$$
\left\|u_{\varepsilon}\right\|_{W^{2, p}(\Omega)} \lesssim\left\|f m\left(\frac{\cdot}{\varepsilon}\right)\right\|_{L^{p}(\Omega)} \lesssim\|f\|_{L^{p}(\Omega)}
$$

with constants independent of $\varepsilon$, where we have used the property (2.4) of the invariant measure in the second inequality.

Let us now assume that $(\Omega, A, f) \in \mathcal{H}^{0}$. Noting that $(2.3)$ implies the Cordes condition for $A(\dot{\bar{\varepsilon}})$ with the same constant $\delta \in(0,1]$ for any $\varepsilon>0$, the proof of Theorem 3 from [35] yields the estimate

$$
\left\|u_{\varepsilon}\right\|_{H^{2}(\Omega)} \leq \frac{C(n, \operatorname{diam}(\Omega))}{1-\sqrt{1-\delta}}\left\|\gamma\left(\frac{\cdot}{\varepsilon}\right)\right\|_{L^{\infty}\left(\mathbb{R}^{n}\right)}\|f\|_{L^{2}(\Omega)},
$$

where $\gamma$ is the function given by

$$
\gamma: \mathbb{R}^{n} \rightarrow \mathbb{R}, \quad \gamma(y):=\frac{\operatorname{tr} A(y)}{|A(y)|^{2}}
$$

We observe that by (2.1), there exist constants $\bar{\gamma}, \Gamma>0$ such that

$$
0<\bar{\gamma} \leq \gamma(y) \leq \Gamma \quad \forall y \in \mathbb{R}^{n} .
$$

Therefore, we obtain from (5.1) the bound

$$
\left\|u_{\varepsilon}\right\|_{H^{2}(\Omega)} \lesssim\|f\|_{L^{2}(\Omega)}
$$

with a constant that is independent of $\varepsilon$.

Proof of Theorem 2.6. By Theorem 2.5, the reflexivity of $W^{2, p}(\Omega)$ for $p \in(1, \infty)$, the compactness of the embedding $W^{2, p}(\Omega) \hookrightarrow W^{1, p}(\Omega)$, and the properties of the trace operator, there exists a $u_{0} \in W^{2, p}(\Omega) \cap W_{0}^{1, p}(\Omega)$ such that (for a subsequence, not indicated,)

$$
\begin{array}{ll}
u_{\varepsilon} \rightarrow u_{0} & \text { weakly in } W^{2, p}(\Omega), \text { and } \\
u_{\varepsilon} \rightarrow u_{0} & \text { strongly in } W^{1, p}(\Omega) .
\end{array}
$$

We can transform (2.2) as in Section 2.2 into the divergence-form problem (2.6) with

$$
A^{\text {div }}=A m+B
$$

being $Y$-periodic, Hölder continuous and uniformly elliptic on $\mathbb{R}^{n}$. Recalling that $B$ is of mean zero over $Y$, we have

$$
A^{\text {div }}\left(\frac{\cdot}{\varepsilon}\right) \stackrel{*}{\rightarrow} \int_{Y} A m=A^{0} \quad \text { weakly-* in } L^{\infty}(\Omega) .
$$

Since we have that

$$
\nabla u_{\varepsilon} \rightarrow \nabla u_{0} \quad \text { strongly in } L^{p}(\Omega)
$$

we can pass to the limit in the weak formulation of (2.6) to obtain that $u_{0} \in W^{2, p}(\Omega) \cap W_{0}^{1, p}(\Omega)$ solves $(2.7)$. We conclude the proof by noting that (2.7) admits a unique strong solution in $W^{2, p}(\Omega) \cap W_{0}^{1, p}(\Omega)$. 
Proof of Theorem 2.7. First, we note that since $A \in C^{0, \alpha}\left(\mathbb{R}^{n}\right)$, we have $\chi_{i j} \in C^{2, \alpha}\left(\mathbb{R}^{n}\right)$ for any $1 \leq i, j \leq n$ by elliptic regularity theory. A direct computation shows that the function

$$
\tilde{u}_{\varepsilon}:=u_{0}+\varepsilon^{2} \sum_{i, j=1}^{n} \chi_{i j}\left(\frac{\cdot}{\varepsilon}\right) \partial_{i j}^{2} u_{0}
$$

solves the problem

$$
\left\{\begin{aligned}
A\left(\frac{\cdot}{\varepsilon}\right): D^{2} \tilde{u}_{\varepsilon} & =f+\varepsilon F_{\varepsilon} & & \text { in } \Omega \\
\tilde{u}_{\varepsilon} & =\varepsilon^{2} \sum_{i, j=1}^{n} \chi_{i j}\left(\frac{\cdot}{\varepsilon}\right) \partial_{i j}^{2} u_{0} & & \text { on } \partial \Omega
\end{aligned}\right.
$$

where

$$
F_{\varepsilon}:=\sum_{i, j, k, l=1}^{n} a_{i j}\left(\frac{\dot{ }}{\varepsilon}\right)\left(2 \partial_{i} \chi_{k l}\left(\frac{\cdot}{\varepsilon}\right) \partial_{j k l}^{3} u_{0}+\varepsilon \chi_{k l}\left(\frac{\cdot}{\varepsilon}\right) \partial_{i j k l}^{4} u_{0}\right) .
$$

Note that since $u_{0} \in W^{4, p}(\Omega)$, one has that

$$
\left\|F_{\varepsilon}\right\|_{L^{p}(\Omega)} \lesssim\left\|u_{0}\right\|_{W^{4, p}(\Omega)},
$$

with the constant being independent of $\varepsilon$. We then have that $d_{\varepsilon}:=\tilde{u}_{\varepsilon}-u_{\varepsilon}$ satisfies

$$
\left\{\begin{aligned}
A\left(\frac{\cdot}{\varepsilon}\right): D^{2} d_{\varepsilon} & =\varepsilon F_{\varepsilon} & & \text { in } \Omega \\
d_{\varepsilon} & =\varepsilon^{2} \sum_{i, j=1}^{n} \chi_{i j}\left(\frac{\cdot}{\varepsilon}\right) \partial_{i j}^{2} u_{0} & & \text { on } \partial \Omega .
\end{aligned}\right.
$$

Therefore, by the definition of the boundary corrector,

$$
\left\{\begin{aligned}
A\left(\frac{\cdot}{\varepsilon}\right): D^{2}\left(d_{\varepsilon}+\varepsilon^{2} \theta_{\varepsilon}\right) & =\varepsilon F_{\varepsilon} & & \text { in } \Omega \\
d_{\varepsilon}+\varepsilon^{2} \theta_{\varepsilon} & =0 & & \text { on } \partial \Omega .
\end{aligned}\right.
$$

We conclude using the estimate from Theorem 2.5 that

$$
\left\|d_{\varepsilon}+\varepsilon^{2} \theta_{\varepsilon}\right\|_{W^{2, p}(\Omega)} \lesssim \varepsilon\left\|F_{\varepsilon}\right\|_{L^{p}(\Omega)} \lesssim \varepsilon\left\|u_{0}\right\|_{W^{4, p}(\Omega)},
$$

and (2.9) holds.

Proof of Theorem 2.8. Let $\eta \in C_{c}^{\infty}\left(\mathbb{R}^{n}\right)$ be a cut-off function with $0 \leq \eta \leq 1$,

$$
\begin{aligned}
& \eta \equiv 1 \quad \text { in }\left\{x \in \Omega: \operatorname{dist}(x, \partial \Omega)<\frac{\varepsilon}{2}\right\} \\
& \eta \equiv 0 \quad \text { in }\{x \in \Omega: \operatorname{dist}(x, \partial \Omega) \geq \varepsilon\}
\end{aligned}
$$

and let $\eta$ satisfy

$$
|\nabla \eta|+\varepsilon\left|D^{2} \eta\right| \lesssim \frac{1}{\varepsilon} \quad \text { in } \Omega
$$


We introduce the function

$$
\tilde{\theta}_{\varepsilon}:=\theta_{\varepsilon}+\eta \sum_{i, j=1}^{n} \chi_{i j}\left(\frac{\dot{-}}{\varepsilon}\right) \partial_{i j}^{2} u_{0}
$$

and verify that

$$
A\left(\frac{\dot{\varepsilon}}{\varepsilon}\right): D^{2} \tilde{\theta}_{\varepsilon}=\sum_{i, j, k, l=1}^{n} a_{i j}\left(\frac{\dot{ }}{\varepsilon}\right) \partial_{i j}^{2}\left(\eta \chi_{k l}\left(\frac{\dot{-}}{\varepsilon}\right) \partial_{k l}^{2} u_{0}\right)=\frac{1}{\varepsilon^{2}} S_{1}+\frac{1}{\varepsilon} S_{2}+S_{3},
$$

where $S_{1}, S_{2}$ and $S_{3}$ are given by

$$
\begin{aligned}
S_{1} & :=\sum_{i, j, k, l=1}^{n} a_{i j}\left(\frac{\dot{-}}{\varepsilon}\right) \eta \partial_{i j}^{2} \chi_{k l}\left(\frac{\cdot}{\varepsilon}\right) \partial_{k l}^{2} u_{0}, \\
S_{2} & :=2 \sum_{i, j, k, l=1}^{n} a_{i j}\left(\frac{\cdot}{\varepsilon}\right)\left(\partial_{i} \eta \partial_{j} \chi_{k l}\left(\frac{\cdot}{\varepsilon}\right) \partial_{k l}^{2} u_{0}+\eta \partial_{i} \chi_{k l}\left(\frac{\cdot}{\varepsilon}\right) \partial_{j k l}^{3} u_{0}\right) \\
S_{3} & :=\sum_{i, j, k, l=1}^{n} a_{i j}\left(\frac{\cdot}{\varepsilon}\right)\left(\partial_{i j}^{2} \eta \chi_{k l}\left(\frac{\cdot}{\varepsilon}\right) \partial_{k l}^{2} u_{0}+2 \partial_{i} \eta \chi_{k l}\left(\frac{\cdot}{\varepsilon}\right) \partial_{j k l}^{3} u_{0}+\eta \chi_{k l}\left(\frac{\dot{\varepsilon}}{\varepsilon}\right) \partial_{i j k l}^{4} u_{0}\right) .
\end{aligned}
$$

Therefore, $\tilde{\theta}_{\varepsilon}$ satisfies

$$
\left\{\begin{aligned}
A\left(\frac{\cdot}{\varepsilon}\right): D^{2} \tilde{\theta}_{\varepsilon} & =\frac{1}{\varepsilon^{2}} S_{1}+\frac{1}{\varepsilon} S_{2}+S_{3} & & \text { in } \Omega, \\
\tilde{\theta}_{\varepsilon} & =0 & & \text { on } \partial \Omega .
\end{aligned}\right.
$$

Since $u_{0} \in W^{4, p}(\Omega) \cap W^{2, \infty}(\Omega)$ by assumption, the right-hand side belongs to $L^{p}(\Omega)$, and we have by Theorem 2.5 that

$$
\left\|\tilde{\theta}_{\varepsilon}\right\|_{W^{2, p}(\Omega)} \lesssim \frac{1}{\varepsilon^{2}}\left\|S_{1}\right\|_{L^{p}(\Omega)}+\frac{1}{\varepsilon}\left\|S_{2}\right\|_{L^{p}(\Omega)}+\left\|S_{3}\right\|_{L^{p}(\Omega)} .
$$

We look at the terms on the right-hand side separately and start with $S_{1}$. Using the boundedness of $A$ and the fact that $\chi_{i j} \in W^{2, \infty}\left(\mathbb{R}^{n}\right)$, we have

$$
\begin{aligned}
\left\|S_{1}\right\|_{L^{p}(\Omega)} & =\left\|\sum_{i, j, k, l=1}^{n} a_{i j}\left(\frac{\dot{-}}{\varepsilon}\right) \eta \partial_{i j}^{2} \chi_{k l}\left(\frac{\dot{-}}{\varepsilon}\right) \partial_{k l}^{2} u_{0}\right\|_{L^{p}(\Omega)} \\
& \lesssim\left\|u_{0}\right\|_{W^{2, \infty}(\Omega)}\|\eta\|_{L^{p}(\Omega)} \\
& \lesssim|\{x \in \Omega: \operatorname{dist}(x, \partial \Omega)<\varepsilon\}|^{\frac{1}{p}}\left\|u_{0}\right\|_{W^{2, \infty}(\Omega)} \\
& \lesssim \varepsilon^{\frac{1}{p}}\left\|u_{0}\right\|_{W^{2, \infty}(\Omega)} .
\end{aligned}
$$

For $S_{2}$, we obtain similarly that

$$
\begin{aligned}
\left\|S_{2}\right\|_{L^{p}(\Omega)} & =\left\|2 \sum_{i, j, k, l=1}^{n} a_{i j}\left(\frac{\cdot}{\varepsilon}\right)\left(\partial_{i} \eta \partial_{j} \chi_{k l}\left(\frac{\cdot}{\varepsilon}\right) \partial_{k l}^{2} u_{0}+\eta \partial_{i} \chi_{k l}\left(\frac{\cdot}{\varepsilon}\right) \partial_{j k l}^{3} u_{0}\right)\right\|_{L^{p}(\Omega)} \\
& \lesssim\|\nabla \eta\|_{L^{p}(\Omega)}\left\|u_{0}\right\|_{W^{2, \infty}(\Omega)}+\|\eta\|_{L^{\infty}(\Omega)}\left\|u_{0}\right\|_{W^{4, p}(\Omega)} \\
& \lesssim \frac{1}{\varepsilon}|\{x \in \Omega: \operatorname{dist}(x, \partial \Omega)<\varepsilon\}|^{\frac{1}{p}}\left\|u_{0}\right\|_{W^{2, \infty}(\Omega)}+\left\|u_{0}\right\|_{W^{4, p}(\Omega)} \\
& \lesssim \frac{1}{\varepsilon^{1-\frac{1}{p}}}\left\|u_{0}\right\|_{W^{2, \infty}(\Omega)}+\left\|u_{0}\right\|_{W^{4, p}(\Omega)} .
\end{aligned}
$$


Finally, for $S_{3}$, we have that

$$
\begin{aligned}
\left\|S_{3}\right\|_{L^{p}(\Omega)} & =\left\|\sum_{i, j, k, l=1}^{n} a_{i j}\left(\frac{\dot{-}}{\varepsilon}\right)\left(\partial_{i j}^{2} \eta \chi_{k l}\left(\frac{\dot{ }}{\varepsilon}\right) \partial_{k l}^{2} u_{0}+2 \partial_{i} \eta \chi_{k l}\left(\frac{\dot{ }}{\varepsilon}\right) \partial_{j k l}^{3} u_{0}+\eta \chi_{k l}\left(\frac{\dot{ }}{\varepsilon}\right) \partial_{i j k l}^{4} u_{0}\right)\right\|_{L^{p}(\Omega)} \\
& \lesssim\left\|D^{2} \eta\right\|_{L^{p}(\Omega)}\left\|u_{0}\right\|_{W^{2, \infty}(\Omega)}+\left(\|\nabla \eta\|_{L^{\infty}(\Omega)}+\|\eta\|_{L^{\infty}(\Omega)}\right)\left\|u_{0}\right\|_{W^{4, p}(\Omega)} \\
& \lesssim \frac{1}{\varepsilon^{2}}|\{x \in \Omega: \operatorname{dist}(x, \partial \Omega)<\varepsilon\}|^{\frac{1}{p}}\left\|u_{0}\right\|_{W^{2, \infty}(\Omega)}+\frac{1}{\varepsilon}\left\|u_{0}\right\|_{W^{4, p}(\Omega)} \\
& \lesssim \frac{1}{\varepsilon^{2-\frac{1}{p}}}\left\|u_{0}\right\|_{W^{2, \infty}(\Omega)}+\frac{1}{\varepsilon}\left\|u_{0}\right\|_{W^{4, p}(\Omega)} .
\end{aligned}
$$

Altogether, we have shown that

$$
\begin{aligned}
\left\|\tilde{\theta}_{\varepsilon}\right\|_{W^{2, p}(\Omega)} & \lesssim\left(\frac{\varepsilon^{\frac{1}{p}}}{\varepsilon^{2}}+\frac{1}{\varepsilon \cdot \varepsilon^{1-\frac{1}{p}}}+\frac{1}{\varepsilon^{2-\frac{1}{p}}}\right)\left\|u_{0}\right\|_{W^{2, \infty}(\Omega)}+\left(\frac{1}{\varepsilon}+\frac{1}{\varepsilon}\right)\left\|u_{0}\right\|_{W^{4, p}(\Omega)} \\
& \lesssim \frac{1}{\varepsilon^{2-\frac{1}{p}}}\left\|u_{0}\right\|_{W^{2, \infty}(\Omega)}+\frac{1}{\varepsilon}\left\|u_{0}\right\|_{W^{4, p}(\Omega)} .
\end{aligned}
$$

By direct computation, using the bounds

$$
\|\eta\|_{L^{p}(\Omega)} \lesssim \varepsilon^{\frac{1}{p}}, \quad\|\nabla \eta\|_{L^{p}(\Omega)} \lesssim \frac{1}{\varepsilon^{1-\frac{1}{p}}}, \quad\left\|D^{2} \eta\right\|_{L^{p}(\Omega)} \lesssim \frac{1}{\varepsilon^{2-\frac{1}{p}}},
$$

we can show that

$$
\left\|\eta \sum_{i, j=1}^{n} \chi_{i j}\left(\frac{\cdot}{\varepsilon}\right) \partial_{i j}^{2} u_{0}\right\|_{W^{2, p}(\Omega)} \lesssim \frac{1}{\varepsilon^{2-\frac{1}{p}}}\left\|u_{0}\right\|_{W^{2, \infty}(\Omega)}+\frac{1}{\varepsilon}\left\|u_{0}\right\|_{W^{4, p}(\Omega)} .
$$

Therefore, using the triangle inequality, we obtain that

$$
\left\|\theta_{\varepsilon}\right\|_{W^{2, p}(\Omega)} \lesssim \frac{1}{\varepsilon^{2-\frac{1}{p}}}\left\|u_{0}\right\|_{W^{2, \infty}(\Omega)}+\frac{1}{\varepsilon}\left\|u_{0}\right\|_{W^{4, p}(\Omega)} .
$$

We conclude that

$$
\left\|\varepsilon^{2} \theta_{\varepsilon}\right\|_{W^{2, p}(\Omega)} \lesssim \varepsilon^{\frac{1}{p}}\left\|u_{0}\right\|_{W^{2, \infty}(\Omega)}+\varepsilon\left\|u_{0}\right\|_{W^{4, p}(\Omega)} .
$$

The claim now follows from (2.9).

\subsection{Proofs of the numerical results}

Proof of Theorem 3.1. We observe that $m=\tilde{m}+1$, where $\tilde{m}$ is the unique solution to the problem

$$
\left\{\begin{array}{l}
-\nabla \cdot(A \nabla \tilde{m}+\tilde{m} \operatorname{div} A)=\nabla \cdot(\operatorname{div} A) \quad \text { in } Y, \\
\tilde{m} \text { is } Y \text {-periodic, } \int_{Y} \tilde{m}=0
\end{array}\right.
$$

that is,

$$
\tilde{m} \in W_{\text {per }}(Y), \quad a(\tilde{m}, \varphi)=-\int_{Y}(\operatorname{div} A) \cdot \nabla \varphi \quad \forall \varphi \in W_{\text {per }}(Y)
$$


where

$$
a: W_{\mathrm{per}}(Y) \times W_{\mathrm{per}}(Y) \longrightarrow \mathbb{R}, \quad a(u, v):=\int_{Y} A \nabla u \cdot \nabla v+\int_{Y} u(\operatorname{div} A) \cdot \nabla v .
$$

We further observe that (3.1) is equivalent to

$$
\tilde{m}_{h} \in \tilde{M}_{h}, \quad a\left(\tilde{m}_{h}, \varphi_{h}\right)=-\int_{Y}(\operatorname{div} A) \cdot \nabla \varphi_{h} \quad \forall \varphi_{h} \in \tilde{M}_{h} .
$$

We start by showing boundedness of $a$ and a Gårding-type inequality. We claim that there exist constants $C_{b}, C_{g}>0$ such that

$$
|a(u, v)| \leq C_{b}\|u\|_{H^{1}(Y)}\|v\|_{H^{1}(Y)} \quad \forall u, v \in W_{\text {per }}(Y),
$$

and

$$
a(u, u) \geq \frac{\lambda}{2}\|u\|_{H^{1}(Y)}^{2}-C_{g}\|u\|_{L^{2}(Y)}^{2} \quad \forall u \in W_{\text {per }}(Y) .
$$

Let us first show (5.3). For $u, v \in W_{\text {per }}(Y)$, by Hölder's inequality and Sobolev embeddings (note that, according to (2.1), $q>n)$, we have that

$$
\left|\int_{Y} u(\operatorname{div} A) \cdot \nabla v\right| \leq\|\operatorname{div} A\|_{L^{q}(Y)}\|u\|_{L^{\frac{2 q}{q-2}}(Y)}\|\nabla v\|_{L^{2}(Y)} \lesssim\|u\|_{H^{1}(Y)}\|v\|_{H^{1}(Y)} .
$$

Using the fact that $A \in W^{1, q}(Y) \hookrightarrow L^{\infty}(Y)$ since $q>n$, we obtain the bound

$$
|a(u, v)| \leq\left|\int_{Y} A \nabla u \cdot \nabla v\right|+\left|\int_{Y} u(\operatorname{div} A) \cdot \nabla v\right| \lesssim\|u\|_{H^{1}(Y)}\|v\|_{H^{1}(Y)}
$$

for any $u, v \in W_{\text {per }}(Y)$, i.e., (5.3) holds.

Let us now show the estimate (5.4). For $u \in W_{\text {per }}(Y)$, by ellipticity and Hölder's inequality, we have

$$
\begin{aligned}
a(u, u) & =\int_{Y} A \nabla u \cdot \nabla u+\int_{Y} u(\operatorname{div} A) \cdot \nabla u \\
& \geq \lambda\|\nabla u\|_{L^{2}(Y)}^{2}-\|\operatorname{div} A\|_{L^{q}(Y)}\|u\|_{L^{\frac{2 q}{q-2}}(Y)}\|\nabla u\|_{L^{2}(Y)} .
\end{aligned}
$$

For the second term we use the Gagliardo-Nirenberg inequality and Young's inequality to obtain

$$
\begin{aligned}
\|\operatorname{div} A\|_{L^{q}(Y)}\|u\|_{L^{\frac{2 q}{q-2}(Y)}}\|\nabla u\|_{L^{2}(Y)} & \leq C(q, n)\|\operatorname{div} A\|_{L^{q}(Y)}\|u\|_{L^{2}(Y)}^{1-\frac{n}{q}}\|\nabla u\|_{L^{2}(Y)}^{1+\frac{n}{q}} \\
& \leq \frac{\lambda}{2}\|\nabla u\|_{L^{2}(Y)}^{2}+C\left(q, n, \lambda,\|\operatorname{div} A\|_{L^{q}(Y)}\right)\|u\|_{L^{2}(Y)}^{2} .
\end{aligned}
$$

Therefore, we have

$$
\begin{aligned}
a(u, u) & \geq \frac{\lambda}{2}\|\nabla u\|_{L^{2}(Y)}^{2}-C\left(q, n, \lambda,\|\operatorname{div} A\|_{L^{q}(Y)}\right)\|u\|_{L^{2}(Y)}^{2} \\
& =\frac{\lambda}{2}\|u\|_{H^{1}(Y)}^{2}-\left(\frac{\lambda}{2}+C\left(q, n, \lambda,\|\operatorname{div} A\|_{L^{q}(Y)}\right)\right)\|u\|_{L^{2}(Y)}^{2}
\end{aligned}
$$

for any $u \in W_{\text {per }}(Y)$, i.e., (5.4) holds with

$$
C_{g}:=\frac{\lambda}{2}+C\left(q, n, \lambda,\|\operatorname{div} A\|_{L^{q}(Y)}\right) .
$$


We use Schatz's method to derive an a priori estimate; see [34]. The proof of the uniqueness of $\tilde{m}_{h} \in \tilde{M}_{h}$ (which implies its existence) proceeds analogously and is therefore omitted.

From our Gårding-type inequality (5.4) we see that (note that $\tilde{m}-\tilde{m}_{h} \in W_{\text {per }}(Y)$ )

$$
\begin{aligned}
\left\|\tilde{m}-\tilde{m}_{h}\right\|_{H^{1}(Y)}-\frac{2 C_{g}}{\lambda}\left\|\tilde{m}-\tilde{m}_{h}\right\|_{L^{2}(Y)} & \leq\left\|\tilde{m}-\tilde{m}_{h}\right\|_{H^{1}(Y)}-\frac{2 C_{g}}{\lambda} \frac{\left\|\tilde{m}-\tilde{m}_{h}\right\|_{L^{2}(Y)}^{2}}{\left\|\tilde{m}-\tilde{m}_{h}\right\|_{H^{1}(Y)}} \\
& \leq \frac{2}{\lambda} \frac{a\left(\tilde{m}-\tilde{m}_{h}, \tilde{m}-\tilde{m}_{h}\right)}{\left\|\tilde{m}-\tilde{m}_{h}\right\|_{H^{1}(Y)}}
\end{aligned}
$$

By Galerkin-orthogonality and boundedness, we have for any $\tilde{v}_{h} \in \tilde{M}_{h}$ that

$$
\frac{a\left(\tilde{m}-\tilde{m}_{h}, \tilde{m}-\tilde{m}_{h}\right)}{\left\|\tilde{m}-\tilde{m}_{h}\right\|_{H^{1}(Y)}}=\frac{a\left(\tilde{m}-\tilde{m}_{h}, \tilde{m}-\tilde{v}_{h}\right)}{\left\|\tilde{m}-\tilde{m}_{h}\right\|_{H^{1}(Y)}} \leq C_{b}\left\|\tilde{m}-\tilde{v}_{h}\right\|_{H^{1}(Y)},
$$

and taking the infimum over all $\tilde{v}_{h} \in \tilde{M}_{h}$, we find that

$$
\frac{a\left(\tilde{m}-\tilde{m}_{h}, \tilde{m}-\tilde{m}_{h}\right)}{\left\|\tilde{m}-\tilde{m}_{h}\right\|_{H^{1}(Y)}} \leq C_{b} \inf _{\tilde{v}_{h} \in \tilde{M}_{h}}\left\|\tilde{m}-\tilde{v}_{h}\right\|_{H^{1}(Y)} .
$$

Combining this estimate with (5.5) yields

$$
\left\|\tilde{m}-\tilde{m}_{h}\right\|_{H^{1}(Y)}-\frac{2 C_{g}}{\lambda}\left\|\tilde{m}-\tilde{m}_{h}\right\|_{L^{2}(Y)} \leq \frac{2 C_{b}}{\lambda} \inf _{v_{h} \in \tilde{M}_{h}}\left\|\tilde{m}-\tilde{v}_{h}\right\|_{H^{1}(Y)} .
$$

Next, we use an Aubin-Nitsche-type duality argument.

Let $\phi \in W_{\text {per }}(Y)$ be the unique solution to

$$
\left\{\begin{array}{l}
-\nabla \cdot(A \nabla \phi)+(\operatorname{div} A) \cdot \nabla \phi=\frac{\tilde{m}-\tilde{m}_{h}}{m} \quad \text { in } Y, \\
\phi \text { is } Y \text {-periodic, } \int_{Y} \phi=0 .
\end{array}\right.
$$

We note that the solvability condition (2.5) is satisfied:

$$
\int_{Y} \frac{\tilde{m}-\tilde{m}_{h}}{m} m=\int_{Y}\left(\tilde{m}-\tilde{m}_{h}\right)=0 .
$$

We have, using the bounds on the invariant measure (2.4), the weak formulation of (5.7) and the symmetry of $A$, that

$$
\begin{aligned}
\frac{1}{M}\left\|\tilde{m}-\tilde{m}_{h}\right\|_{L^{2}(Y)}^{2} & \leq \int_{Y} \frac{\tilde{m}-\tilde{m}_{h}}{m}\left(\tilde{m}-\tilde{m}_{h}\right) \\
& =\int_{Y} A \nabla \phi \cdot \nabla\left(\tilde{m}-\tilde{m}_{h}\right)+\int_{Y}(\operatorname{div} A) \cdot \nabla \phi\left(\tilde{m}-\tilde{m}_{h}\right) \\
& =\int_{Y} A \nabla\left(\tilde{m}-\tilde{m}_{h}\right) \cdot \nabla \phi+\int_{Y}\left(\tilde{m}-\tilde{m}_{h}\right)(\operatorname{div} A) \cdot \nabla \phi
\end{aligned}
$$

Next, we use Galerkin orthogonality, the boundedness (5.3) and an interpolation inequality to obtain

$$
\begin{aligned}
\frac{1}{M}\left\|\tilde{m}-\tilde{m}_{h}\right\|_{L^{2}(Y)}^{2} & \leq a\left(\tilde{m}-\tilde{m}_{h}, \phi\right) \\
& =a\left(\tilde{m}-\tilde{m}_{h}, \phi-\mathcal{I}_{h} \phi\right) \\
& \lesssim\left\|\tilde{m}-\tilde{m}_{h}\right\|_{H^{1}(Y)}\left\|\phi-\mathcal{I}_{h} \phi\right\|_{H^{1}(Y)} \\
& \lesssim h\left\|\tilde{m}-\tilde{m}_{h}\right\|_{H^{1}(Y)}\|\phi\|_{H^{2}(Y)},
\end{aligned}
$$


where $\mathcal{I}_{h} \phi$ denotes the continuous piecewise linear interpolant (for $n \leq 3$ and quasi-interpolant for $n \geq 4$ ) of $\phi$ on the triangulation. Finally, by a regularity estimate for $\phi$ and the bounds on the invariant measure (2.4), we arrive at the bound

$$
\|\phi\|_{H^{2}(Y)} \lesssim\left\|\frac{\tilde{m}-\tilde{m}_{h}}{m}\right\|_{L^{2}(Y)} \lesssim\left\|\tilde{m}-\tilde{m}_{h}\right\|_{L^{2}(Y)}
$$

which provides us with the estimate

$$
\left\|\tilde{m}-\tilde{m}_{h}\right\|_{L^{2}(Y)} \leq C_{0} h\left\|\tilde{m}-\tilde{m}_{h}\right\|_{H^{1}(Y)}
$$

for some $C_{0}>0$. Combining this with (5.6) we have

$$
\begin{aligned}
\left(1-\frac{2 C_{g} C_{0}}{\lambda} h\right)\left\|\tilde{m}-\tilde{m}_{h}\right\|_{H^{1}(Y)} & \leq\left\|\tilde{m}-\tilde{m}_{h}\right\|_{H^{1}(Y)}-\frac{2 C_{g}}{\lambda}\left\|\tilde{m}-\tilde{m}_{h}\right\|_{L^{2}(Y)} \\
& \leq \frac{2 C_{b}}{\lambda} \inf _{\tilde{v}_{h} \in \tilde{M}_{h}}\left\|\tilde{m}-\tilde{v}_{h}\right\|_{H^{1}(Y)} .
\end{aligned}
$$

Therefore, for $h$ sufficiently small, we arrive at the bounds

$$
\left\|\tilde{m}-\tilde{m}_{h}\right\|_{H^{1}(Y)} \lesssim \inf _{\tilde{v}_{h} \in \tilde{M}_{h}}\left\|\tilde{m}-\tilde{v}_{h}\right\|_{H^{1}(Y)}
$$

and

$$
\left\|\tilde{m}-\tilde{m}_{h}\right\|_{L^{2}(Y)} \leq C_{0} h\left\|\tilde{m}-\tilde{m}_{h}\right\|_{H^{1}(Y)} \lesssim h \inf _{\tilde{v}_{h} \in \tilde{M}_{h}}\left\|\tilde{m}-\tilde{v}_{h}\right\|_{H^{1}(Y)} .
$$

We have thus established the a priori estimate

$$
\left\|\tilde{m}-\tilde{m}_{h}\right\|_{L^{2}(Y)}+h\left\|\tilde{m}-\tilde{m}_{h}\right\|_{H^{1}(Y)} \lesssim h \inf _{\tilde{v}_{h} \in \tilde{M}_{h}}\left\|\tilde{m}-\tilde{v}_{h}\right\|_{H^{1}(Y)} .
$$

Finally, using that $m=\tilde{m}+1$ and $m_{h}=\tilde{m}_{h}+1$, we conclude that

$$
\left\|m-m_{h}\right\|_{L^{2}(Y)}+h\left\|m-m_{h}\right\|_{H^{1}(Y)} \lesssim h \inf _{\tilde{v}_{h} \in \tilde{M}_{h}}\left\|m-\left(\tilde{v}_{h}+1\right)\right\|_{H^{1}(Y)} .
$$

Proof of Lemma 3.3. Fix $1 \leq i, j \leq n$. Using the definition of $A^{0}=\left(a_{i j}^{0}\right)$, i.e.,

$$
a_{i j}^{0}=\int_{Y} a_{i j} m
$$

we obtain the estimate

$$
\left|a_{i j}^{0}-a_{i j, h}^{0}\right| \leq\left\|a_{i j}\left(m-m_{h}\right)\right\|_{L^{1}(Y)}+\left\|a_{i j} m_{h}-\mathcal{I}_{h}\left(a_{i j} m_{h}\right)\right\|_{L^{1}(Y)} .
$$

For the first term, we have

$$
\left\|a_{i j}\left(m-m_{h}\right)\right\|_{L^{1}(Y)} \lesssim\left\|m-m_{h}\right\|_{L^{1}(Y)} \lesssim\left\|m-m_{h}\right\|_{L^{2}(Y)} .
$$

For the second term, let us first note that using $a_{i j} \in W^{1, q}(Y)$ with $q>n$ and Sobolev embeddings, we have

$$
\begin{aligned}
\left|a_{i j} m_{h}\right|_{H^{1}(Y)} & \leq\left\|\nabla a_{i j}\right\|_{L^{q}(Y)}\left\|m_{h}\right\|_{L^{\frac{2 q}{q-2}(Y)}}+\left\|a_{i j}\right\|_{L^{\infty}(Y)}\left\|\nabla m_{h}\right\|_{L^{2}(Y)} \\
& \lesssim\left\|a_{i j}\right\|_{W^{1, q}(Y)}\left\|m_{h}\right\|_{H^{1}(Y)} .
\end{aligned}
$$


Therefore, using a standard interpolation error bound, we obtain

$$
\begin{aligned}
\left\|a_{i j} m_{h}-\mathcal{I}_{h}\left(a_{i j} m_{h}\right)\right\|_{L^{1}(Y)} & \lesssim\left\|a_{i j} m_{h}-\mathcal{I}_{h}\left(a_{i j} m_{h}\right)\right\|_{L^{2}(Y)} \\
& \lesssim h\left|a_{i j} m_{h}\right|_{H^{1}(Y)} \\
& \lesssim h\left\|a_{i j}\right\|_{W^{1, q}(Y)}\left\|m_{h}\right\|_{H^{1}(Y)} .
\end{aligned}
$$

By Theorem 3.1, for $h>0$ sufficiently small, we have that

$$
\begin{aligned}
\left|a_{i j}^{0}-a_{i j, h}^{0}\right| & \lesssim\left\|m-m_{h}\right\|_{L^{2}(Y)}+h\left\|m_{h}\right\|_{H^{1}(Y)} \\
& \lesssim\left\|m-m_{h}\right\|_{L^{2}(Y)}+h\left\|m-m_{h}\right\|_{H^{1}(Y)}+h\|m\|_{H^{1}(Y)} \\
& \lesssim h \inf _{\tilde{v}_{h} \in \tilde{M}_{h}}\left\|m-\left(\tilde{v}_{h}+1\right)\right\|_{H^{1}(Y)}+h\|m\|_{H^{1}(Y)} \\
& \lesssim h\|m-1\|_{H^{1}(Y)}+h\|m\|_{H^{1}(Y)} \\
& \lesssim h .
\end{aligned}
$$

Finally, we note that this implies that for $h>0$ sufficiently small, $A_{h}^{0}$ is elliptic.

Proof of Lemma 3.4. We let $w_{h}:=u_{0}-u_{0}^{h} \in H^{2}(\Omega) \cap H_{0}^{1}(\Omega)$ and note that $w_{h}$ is the unique solution to the boundary-value problem

$$
\left\{\begin{aligned}
A^{0}: D^{2} w_{h} & =\left(A_{h}^{0}-A^{0}\right): D^{2} u_{0}^{h} & & \text { in } \Omega, \\
w_{h} & =0 & & \text { on } \partial \Omega .
\end{aligned}\right.
$$

We recall that $A^{0} \in \mathbb{R}^{n \times n}$ is an elliptic constant matrix. For $h>0$ sufficiently small, by an $H^{2}$ a priori estimate, the Cauchy-Schwarz inequality and Lemma 3.3,

$$
\begin{aligned}
\left\|w_{h}\right\|_{H^{2}(\Omega)} & \lesssim\left\|\left(A_{h}^{0}-A^{0}\right): D^{2} u_{0}^{h}\right\|_{L^{2}(\Omega)} \\
& \lesssim\left(\int_{\Omega}\left|\sum_{i, j=1}^{n}\left(a_{i j, h}^{0}-a_{i j}^{0}\right) \partial_{i j}^{2} u_{0}^{h}\right|^{2}\right)^{\frac{1}{2}} \\
& \lesssim\left(\int_{\Omega}\left(\sum_{i, j=1}^{n}\left|a_{i j, h}^{0}-a_{i j}^{0}\right|^{2}\right)\left(\sum_{i, j=1}^{n}\left|\partial_{i j}^{2} u_{0}^{h}\right|^{2}\right)\right)^{\frac{1}{2}} \\
& \lesssim h\left|u_{0}^{h}\right|_{H^{2}(\Omega)} .
\end{aligned}
$$

Finally, we show that for $h>0$ sufficiently small, we have

$$
\left\|u_{0}^{h}\right\|_{H^{2}(\Omega)} \lesssim\|f\|_{L^{2}(\Omega)}
$$

with the constant being independent of $h$. This can be seen by rewriting (3.2) as

$$
\left\{\begin{aligned}
A^{0}: D^{2} u_{0}^{h} & =f+\left(A^{0}-A_{h}^{0}\right): D^{2} u_{0}^{h} & & \text { in } \Omega, \\
u_{0}^{h} & =0 & & \text { on } \partial \Omega .
\end{aligned}\right.
$$

Then, again by an $H^{2}$ a priori estimate and Lemma 3.3,

$$
\left\|u_{0}^{h}\right\|_{H^{2}(\Omega)} \lesssim\left\|f+\left(A^{0}-A_{h}^{0}\right): D^{2} u_{0}^{h}\right\|_{L^{2}(\Omega)} \lesssim\|f\|_{L^{2}(\Omega)}+h\left\|u_{0}^{h}\right\|_{H^{2}(\Omega)}
$$

with constants independent of $h$, i.e., for $h>0$ sufficiently small, (5.8) holds with the constant being independent of $h$. 
Proof of Theorem 3.11. We use (3.9) and the triangle inequality to obtain

$$
\begin{aligned}
\left\|u_{\varepsilon}-u_{0, h}\right\|_{H^{1}(\Omega)} & \leq\left\|u_{\varepsilon}-u_{0}\right\|_{H^{1}(\Omega)}+\left\|u_{0}-u_{0, h}\right\|_{H^{1}(\Omega)} \\
& \lesssim \sqrt{\varepsilon}\left\|u_{0}\right\|_{W^{2, \infty}(\Omega)}+\varepsilon\left\|u_{0}\right\|_{H^{4}(\Omega)}+h\|f\|_{H^{1}(\Omega)},
\end{aligned}
$$

and for $1 \leq k, l \leq n$,

$$
\begin{aligned}
\left\|\partial_{k l}^{2} u_{\varepsilon}-u_{\varepsilon, h}^{k l}\right\|_{L^{1}(\Omega)} \lesssim & \sqrt{\varepsilon}\left\|u_{0}\right\|_{W^{2, \infty}(\Omega)}+\varepsilon\left\|u_{0}\right\|_{H^{4}(\Omega)}+h\|f\|_{H^{1}(\Omega)} \\
& +\sum_{i, j=1}^{n}\left\|\left(\partial_{k l}^{2} \chi_{i j}\right)\left(\frac{\cdot}{\varepsilon}\right) \partial_{i j}^{2} u_{0}-z_{i j, h}^{k l}\left(\frac{\cdot}{\varepsilon}\right) \partial_{i j}^{2} u_{0, h}\right\|_{L^{1}(\Omega)} .
\end{aligned}
$$

It remains to study the last term on the right-hand side of the above inequality. For fixed $1 \leq i, j \leq n$, we use again the triangle inequality to obtain

$$
\begin{aligned}
\left\|\left(\partial_{k l}^{2} \chi_{i j}\right)\left(\frac{\cdot}{\varepsilon}\right) \partial_{i j}^{2} u_{0}-z_{i j, h}^{k l}\left(\frac{\cdot}{\varepsilon}\right) \partial_{i j}^{2} u_{0, h}\right\|_{L^{1}(\Omega)} \\
\quad \leq\left\|z_{i j, h}^{k l}\left(\frac{\dot{-}}{\varepsilon}\right)\left(\partial_{i j}^{2} u_{0}-\partial_{i j}^{2} u_{0, h}\right)\right\|_{L^{1}(\Omega)}+\left\|\left(\partial_{k l}^{2} \chi_{i j}-z_{i j, h}^{k l}\right)\left(\frac{\dot{-}}{\varepsilon}\right) \partial_{i j}^{2} u_{0}\right\|_{L^{1}(\Omega)} \\
\quad \lesssim\left\|z_{i j, h}^{k l}\left(\frac{\dot{\varepsilon}}{\varepsilon}\right)\right\|_{L^{2}(\Omega)}\left\|u_{0}-u_{0, h}\right\|_{H^{2}(\Omega)}+\left\|\left(\partial_{k l}^{2} \chi_{i j}-z_{i j, h}^{k l}\right)(\dot{\dot{\varepsilon}})\right\|_{L^{2}(\Omega)}\left\|u_{0}\right\|_{W^{2, \infty}(\Omega)} \\
\quad \lesssim h\left(\left\|z_{i j, h}^{k l}\left(\frac{\dot{-}}{\varepsilon}\right)\right\|_{L^{2}(\Omega)}\|f\|_{H^{1}(\Omega)}+\left\|u_{0}\right\|_{W^{2, \infty}(\Omega)}\right) .
\end{aligned}
$$

In the last step, we used that by the transformation formula and periodicity (cover $\Omega / \varepsilon$ by $\mathcal{O}\left(\varepsilon^{-n}\right)$ many cells of unit length), there holds

$$
\left\|\left(\partial_{k l}^{2} \chi_{i j}-z_{i j, h}^{k l}\right)\left(\frac{\cdot}{\varepsilon}\right)\right\|_{L^{2}(\Omega)} \lesssim\left\|\partial_{k l}^{2} \chi_{i j}-z_{i j, h}^{k l}\right\|_{L^{2}(Y)} \lesssim h .
$$

We claim that

$$
\left\|z_{i j, h}^{k l}\left(\frac{\dot{\varepsilon}}{\varepsilon}\right)\right\|_{L^{2}(\Omega)} \lesssim h+1
$$

Indeed, we use the triangle inequality, (5.10) and the fact that $\chi_{i j} \in W^{2, \infty}(Y)$ to obtain

$$
\left\|z_{i j, h}^{k l}\left(\frac{\dot{ }}{\varepsilon}\right)\right\|_{L^{2}(\Omega)} \leq\left\|\left(\partial_{k l}^{2} \chi_{i j}-z_{i j, h}^{k l}\right)\left(\frac{\cdot}{\varepsilon}\right)\right\|_{L^{2}(\Omega)}+\left\|\partial_{k l}^{2} \chi_{i j}\right\|_{L^{\infty}(Y)} \lesssim h+1 .
$$

Proof of Theorem 3.15. (i) For $(\Omega, A, f) \in \mathcal{H}$, one shows similarly to the proof of Theorem 3 from [35] and Theorem 2.5 that

$$
\left\|u_{\varepsilon}\right\|_{H^{2}(\Omega)} \lesssim\left\|\frac{\operatorname{tr} A(\cdot, \dot{\bar{\varepsilon}})}{|A(\cdot, \dot{\bar{\varepsilon}})|^{2}}\right\|_{L^{\infty}(\Omega)}\|f\|_{L^{2}(\Omega)} \lesssim\|f\|_{L^{2}(\Omega)} .
$$

For $(\Omega, A, f) \in \mathcal{G}$, the claim follows from the method of freezing coefficients, using the uniform estimate from Theorem 2.5 for the operators $L_{x_{0}}:=A\left(x_{0}, \dot{\bar{\varepsilon}}\right): D^{2}$ for fixed $x_{0} \in \Omega$.

(ii) The uniform estimate from $(i)$ yields weak convergence in $H^{2}(\Omega)$ and strong convergence in $H^{1}(\Omega)$ for a subsequence of $\left(u_{\varepsilon}\right)_{\varepsilon>0}$ to some limit function $u_{0} \in H^{2}(\Omega) \cap H_{0}^{1}(\Omega)$. We multiply (3.10) by $m(\cdot, \dot{\bar{\varepsilon}})$ and follow the transformation performed in [13] to find that the equality

$$
m^{\varepsilon} f=2 \nabla \cdot\left(\tilde{A}^{\varepsilon} \nabla u_{\varepsilon}+\left[\operatorname{div}_{x} \tilde{A}\right]^{\varepsilon} u_{\varepsilon}\right)-2\left[\operatorname{div}_{x} \tilde{A}\right]^{\varepsilon} \cdot \nabla u_{\varepsilon}-\left[D_{x}^{2}: \tilde{A}\right]^{\varepsilon} u_{\varepsilon}-D^{2}:\left(\tilde{A}^{\varepsilon} u_{\varepsilon}\right)
$$


holds weakly, where $\tilde{A}:=A m$ and $v^{\varepsilon}$ denotes $v(\cdot, \dot{\bar{\varepsilon}})$. Passing to the limit, we obtain that $u_{0}$ is a weak solution of (3.13). We conclude the proof by noting that (3.13) admits a unique strong solution, since $A^{0}$ is uniformly elliptic and Lipschitz continuous on $\bar{\Omega}$; see $[25,26]$.

(iii) This can be proved similarly to Theorems 2.7 and 2.8, using that, by the assumptions made on $A$ and elliptic regularity, we have

$$
\chi_{k l}^{\varepsilon},\left[\partial_{x_{i}} \chi_{k l}\right]^{\varepsilon},\left[\partial_{y_{i}} \chi_{k l}\right]^{\varepsilon},\left[\partial_{x_{i} y_{j}}^{2} \chi_{k l}\right]^{\varepsilon},\left[\partial_{x_{i} x_{j}}^{2} \chi_{k l}\right]^{\varepsilon} \in L^{\infty}(\Omega)
$$

for any $1 \leq i, j, k, l \leq n$.

\subsection{Proofs of technical lemmata}

Proof of Lemma 3.6. We start with the case (i). To this end, let $(\Omega, A, f) \in \mathcal{G}^{1,2}$ with $\partial \Omega \in C^{3}$. Then, by elliptic regularity theory, we have $u_{0}^{h} \in H^{3}(\Omega)$. Using elliptic regularity for problem (5.9) yields

$$
\left\|u_{0}^{h}\right\|_{H^{3}(\Omega)} \lesssim\left\|f+\left(A^{0}-A_{h}^{0}\right): D^{2} u_{0}^{h}\right\|_{H^{1}(\Omega)} \lesssim\|f\|_{H^{1}(\Omega)}+h\left\|u_{0}^{h}\right\|_{H^{3}(\Omega)}
$$

with constants independent of $h$, i.e., for $h>0$ sufficiently small, (3.3) holds with the constant being independent of $h$.

Let us now show the claim for the case (ii). To this end, let $(\Omega, A, f) \in \mathcal{H}^{1}$ with $\Omega \subset \mathbb{R}^{2}$ being a polygon and $f \in H_{0}^{1}(\Omega)$. Since

$$
A_{h}^{0}=A^{0}+\left(A_{h}^{0}-A^{0}\right)=: A^{0}+B_{h}
$$

is symmetric and elliptic for $h>0$ sufficiently small, there exists an orthogonal matrix $Q_{h} \in \mathbb{R}^{2 \times 2}$ with $Q_{h} Q_{h}^{\mathrm{T}}=Q_{h}^{\mathrm{T}} Q_{h}=I_{2}$ such that

$$
Q_{h}\left(A^{0}+B_{h}\right) Q_{h}^{\mathrm{T}}=\operatorname{diag}\left(\lambda_{h}^{+}, \lambda_{h}^{-}\right)=: \Lambda_{h}
$$

where $\lambda_{h}^{ \pm}>0$ are given by

$$
2 \lambda_{h}^{ \pm}=\operatorname{tr}\left(A^{0}+B_{h}\right) \pm\left(\left(\operatorname{tr}\left(A^{0}+B_{h}\right)\right)^{2}-4 \operatorname{det}\left(A^{0}+B_{h}\right)\right)^{\frac{1}{2}} .
$$

We note that, by Lemma 3.3, the entries of $B_{h}=\left(b_{i j}^{h}\right)_{1 \leq i, j \leq 2}$ satisfy $b_{i j}^{h} \lesssim h$, and therefore, for $h>0$ sufficiently small, we have $0<\lambda_{h}^{ \pm}+\left(\lambda_{h}^{ \pm}\right)^{-1} \lesssim 1$.

The problem (3.2) in the new coordinates reads

$$
\left\{\begin{array}{cl}
\Delta U_{h}=F_{h} & \text { in } \quad P_{h} \\
U_{h}=0 & \text { on } \quad \partial P_{h},
\end{array}\right.
$$

where $U_{h}:=u_{0}^{h}\left(Q_{h}^{\mathrm{T}} \Lambda_{h}^{\frac{1}{2}} \cdot\right), F_{h}:=f\left(Q_{h}^{\mathrm{T}} \Lambda_{h}^{\frac{1}{2}} \cdot\right)$, and $P_{h}:=\Lambda_{h}^{-\frac{1}{2}} Q_{h} \Omega$. Note that $P_{h}$ is still a bounded convex polygonal domain and that $F_{h} \in H_{0}^{1}\left(P_{h}\right)$. By the change of variables formula and the orthogonality of $Q_{h}$,

$$
\begin{aligned}
\|f\|_{H^{1}(\Omega)}^{2}=\int_{\Omega}\left(|f|^{2}+|\nabla f|^{2}\right) & =\operatorname{det} \Lambda_{h}^{\frac{1}{2}} \int_{P_{h}}\left(\left|f\left(Q_{h}^{\mathrm{T}} \Lambda_{h}^{\frac{1}{2}} \cdot\right)\right|^{2}+\left|\nabla f\left(Q_{h}^{\mathrm{T}} \Lambda_{h}^{\frac{1}{2}} \cdot\right)\right|^{2}\right) \\
& =\operatorname{det} \Lambda_{h}^{\frac{1}{2}} \int_{P_{h}}\left(\left|F_{h}\right|^{2}+\left|Q_{h}^{\mathrm{T}} \Lambda_{h}^{-\frac{1}{2}} \nabla F_{h}\right|^{2}\right) \\
& =\operatorname{det} \Lambda_{h}^{\frac{1}{2}} \int_{P_{h}}\left(\left|F_{h}\right|^{2}+\left|\Lambda_{h}^{-\frac{1}{2}} \nabla F_{h}\right|^{2}\right) \\
& \gtrsim \int_{P_{h}}\left(\left|F_{h}\right|^{2}+\left|\nabla F_{h}\right|^{2}\right)=\left\|F_{h}\right\|_{H^{1}\left(P_{h}\right)}^{2} .
\end{aligned}
$$


Using Lemma 3.7, we have that, for $h>0$ sufficiently small, the solution to (5.11) satisfies

$$
\left\|U_{h}\right\|_{H^{3}\left(P_{h}\right)} \lesssim\left\|F_{h}\right\|_{H^{1}\left(P_{h}\right)} \lesssim\|f\|_{H^{1}(\Omega)}
$$

with constants independent of $h$. It remains to show the bound

$$
\left\|u_{0}^{h}\right\|_{H^{3}(\Omega)} \lesssim\left\|U_{h}\right\|_{H^{3}\left(P_{h}\right)} .
$$

By the change of variables formula and the orthogonality of $Q_{h}$, we obtain similarly as before,

$$
\begin{aligned}
\left\|u_{0}^{h}\right\|_{H^{3}(\Omega)}^{2}= & \int_{\Omega}\left(\left|u_{0}^{h}\right|^{2}+\left|\nabla u_{0}^{h}\right|^{2}+\left|D^{2} u_{0}^{h}\right|^{2}\right)+\sum_{i=1}^{2} \int_{\Omega}\left|D^{2} \partial_{i} u_{0}^{h}\right|^{2} \\
= & \operatorname{det} \Lambda_{h}^{\frac{1}{2}} \int_{P_{h}}\left(\left|U_{h}\right|^{2}+\left|Q_{h}^{\mathrm{T}} \Lambda_{h}^{-\frac{1}{2}} \nabla U_{h}\right|^{2}+\left|Q_{h}^{\mathrm{T}} \Lambda_{h}^{-\frac{1}{2}} D^{2} U_{h} \Lambda_{h}^{-\frac{1}{2}} Q_{h}\right|^{2}\right) \\
& +\sum_{i=1}^{2} \operatorname{det} \Lambda_{h}^{\frac{1}{2}} \int_{P_{h}}\left|\sum_{j=1}^{2} \frac{\left(Q_{h}\right)_{j i}}{\sqrt{\left(\Lambda_{h}\right)_{j j}}} Q_{h}^{\mathrm{T}} \Lambda_{h}^{-\frac{1}{2}} D^{2} \partial_{j} U_{h} \Lambda_{h}^{-\frac{1}{2}} Q_{h}\right|^{2} \\
= & \operatorname{det} \Lambda_{h}^{\frac{1}{2}} \int_{P_{h}}\left(\left|U_{h}\right|^{2}+\left|\Lambda_{h}^{-\frac{1}{2}} \nabla U_{h}\right|^{2}+\left|\Lambda_{h}^{-\frac{1}{2}} D^{2} U_{h} \Lambda_{h}^{-\frac{1}{2}}\right|^{2}\right)+\sum_{i=1}^{2} \frac{\operatorname{det} \Lambda_{h}^{\frac{1}{2}}}{\left(\Lambda_{h}\right)_{i i}} \int_{P_{h}}\left|\Lambda_{h}^{-\frac{1}{2}} D^{2} \partial_{i} U_{h} \Lambda_{h}^{-\frac{1}{2}}\right|^{2} \\
\lesssim & \int_{P_{h}}\left(\left|U_{h}\right|^{2}+\left|\nabla U_{h}\right|^{2}+\left|D^{2} U_{h}\right|^{2}\right)+\sum_{i=1}^{2} \int_{P_{h}}\left|D^{2} \partial_{i} U_{h}\right|^{2}=\left\|U_{h}\right\|_{H^{3}\left(P_{h}\right)}^{2},
\end{aligned}
$$

i.e., we have established the bound (5.12). We conclude that, for $h>0$ sufficiently small, we have (3.3), i.e.,

$$
\left\|u_{0}^{h}\right\|_{H^{3}(\Omega)} \lesssim\|f\|_{H^{1}(\Omega)},
$$

where the constant is independent of $h$.

Proof of Lemma 3.7. First, note that since $\Omega \subset \mathbb{R}^{2}$ is a convex polygonal domain, we have $u \in H^{2}(\Omega) \cap H_{0}^{1}(\Omega)$ with $\|u\|_{H^{2}(\Omega)} \lesssim\|f\|_{L^{2}(\Omega)}$; see [26]. Since $f \in H_{0}^{1}(\Omega)$, there exists a sequence of smooth functions with compact support $\left(f_{m}\right)_{m} \subset C_{c}^{\infty}(\Omega)$ such that $f_{m} \rightarrow f$ in $H^{1}(\Omega)$. Let $\left(u_{m}\right)_{m} \subset H_{0}^{1}(\Omega)$ be the sequence of solutions in $H_{0}^{1}(\Omega)$ to $\Delta u_{m}=f_{m}$ in $\Omega$, and note that $\left(u_{m}\right)_{m} \subset C^{\infty}(\bar{\Omega})$ since the functions $f_{m}$ satisfy compatibility conditions of any order; see Section 5.1 of [26]. Again we use the $H^{2}$-regularity result for solutions of Poisson's problem on convex polygons to obtain

$$
\left\|u_{m}-u\right\|_{H^{2}(\Omega)} \lesssim\left\|f_{m}-f\right\|_{L^{2}(\Omega)} \rightarrow 0
$$

i.e., $u_{m} \rightarrow u$ in $H^{2}(\Omega)$.

Next, we shall use the fact that

$$
|v|_{H^{3}(\Omega)}=\|\nabla(\Delta v)\|_{L^{2}(\Omega)} \quad \forall v \in\left\{w \in H_{0}^{1}(\Omega): \Delta w \in H_{0}^{1}(\Omega)\right\} \cap C^{\infty}(\bar{\Omega}) ;
$$

see [32]. We apply (5.13) to the difference of two elements of the sequence $\left(u_{m}\right)_{m}$ to find that $\left(u_{m}\right)_{m}$ is a Cauchy sequence in $H^{3}(\Omega)$, using that $f_{m} \rightarrow f$ in $H^{1}(\Omega)$. Thus, $u_{m} \rightarrow u$ in $H^{3}(\Omega)$ and passing to the limit in (5.13) applied to the functions $u_{m}$ yields

$$
|u|_{H^{3}(\Omega)}=\|\nabla f\|_{L^{2}(\Omega)} .
$$

Since $\|u\|_{H^{2}(\Omega)} \lesssim\|f\|_{L^{2}(\Omega)}$, we conclude the bound (3.4). 


\section{Conclusion}

In this paper we introduced a scheme for the numerical approximation of elliptic problems in nondivergenceform with rapidly oscillating coefficients on $C^{2, \gamma}$ and polygonal domains, which is based on a $W^{2, p}$ corrector estimate for such problems derived in the first part of this work.

We proved an optimal-order error bound for a finite element approximation of the corresponding invariant measure using continuous $Y$-periodic piecewise linear basis functions on a shape-regular triangulation of the unit cell $Y$ under weak regularity assumptions on the coefficients. The coefficients are integrated against the so obtained approximation of the invariant measure after piecewise linear interpolation on the mesh to obtain an approximation of the constant coefficient-matrix of the homogenized problem. Using an $H^{2}$ comparison result for the solution of this perturbed problem, we eventually obtained an approximation of the solution $u_{0}$ to the homogenized problem in the $H^{2}$-norm. In the case of a polygonal domain in two space dimensions, we made use of compatibility conditions for the source term to ensure sufficiently high Sobolev-regularity of $u_{0}$.

We obtained an approximation to the solution $u_{\varepsilon}$ of the original problem, i.e., the problem with oscillating coefficients, by making use of the $H^{2}$ approximation of $u_{0}$, finite element approximations to second-order derivatives of the corrector functions, as well as an $H^{2}$ corrector result. A method of successively approximating higher derivatives for the approximation of corrector functions was provided and analyzed. The corrector functions are necessary in order to obtain an approximation of $D^{2} u_{\varepsilon}$ whereas the task of approximating $u_{\varepsilon}$ in the $H^{1}$-norm can be achieved using only an $H^{1}$ approximation of $u_{0}$.

Furthermore, we generalized our results to the case of nonuniformly oscillating coefficients, i.e., we derived an analogous corrector result and studied the approximation of the solution $u_{0}$ to the homogenized problem and the solution $u_{\varepsilon}$ of the $\varepsilon$-dependent problem in this case.

In the final part of the paper, we presented numerical experiments matching the theoretical results for problems with both known and unknown $u_{0}$, as well as problems with nonuniformly oscillating coefficients. We illustrated the performance of the scheme for the approximation of the invariant measure, the solution $u_{0}$ to the homogenized problem and the solution $u_{\varepsilon}$ to the problem involving oscillating coefficients for a fixed value of $\varepsilon$.

Future work will focus on weakening of the regularity assumptions on the coefficients and the approximation of fully nonlinear nondivergence-form problems with oscillating coefficients such as the Hamilton-Jacobi-Bellman equation.

Acknowledgements. This work was supported by the UK Engineering and Physical Sciences Research Council [EP/L015811/1]. The authors thank the anonymous referees for their helpful comments and constructive suggestions.

\section{REFERENCES}

[1] A. Abdulle, On a priori error analysis of fully discrete heterogeneous multiscale FEM. Multiscale Model. Simul. 4 (2005) $447-459$.

[2] A. Abdulle, The finite element heterogeneous multiscale method: a computational strategy for multiscale PDEs. Multiple Scales Problems in Biomathematics, Mechanics, Physics and Numerics. In: Vol. 31 of GAKUTO Internat. Ser. Math. Sci. Appl. Gakkotosho, Tokyo (2009) 133-181.

[3] A. Abdulle and M.E. Huber, Finite element heterogeneous multiscale method for nonlinear monotone parabolic homogenization problems. ESAIM: M2AN 50 (2016) 1659-1697.

[4] A. Abdulle and T. Pouchon, A priori error analysis of the finite element heterogeneous multiscale method for the wave equation over long time. SIAM J. Numer. Anal. 54 (2016) 1507-1534.

[5] A. Abdulle, W. E, B. Engquist and E. Vanden-Eijnden, The heterogeneous multiscale method. Acta Numer. 21 (2012) 1-87.

[6] G. Allaire, Shape optimization by the homogenization method. In: Vol. 146 of Applied Mathematical Sciences. Springer-Verlag, New York (2002).

[7] G. Allaire and R. Orive, Homogenization of periodic non self-adjoint problems with large drift and potential. ESAIM: COCV 13 (2007) 735-749.

[8] G. Allaire and A.-L. Raphael, Homogenization of a convection-diffusion model with reaction in a porous medium. C. R. Math. Acad. Sci. Paris 344 (2007) 523-528. 
[9] D. Arjmand and G. Kreiss, An equation-free approach for second order multiscale hyperbolic problems in non-divergence form. Commun. Math. Sci. 16 (2018) 2317-2343.

[10] M. Avellaneda and F.-H. Lin, Compactness methods in the theory of homogenization. II. Equations in nondivergence form. Comm. Pure Appl. Math. 42 (1989) 139-172.

[11] M. Avellaneda and F.-H. Lin, $L^{p}$ bounds on singular integrals in homogenization. Comm. Pure Appl. Math. 44 (1991) 897-910.

[12] I. Babuška, Computation of derivatives in the finite element method. Comment. Math. Univ. Carolinae 11 (1970) 545-558.

[13] A. Bensoussan, L. Boccardo and F. Murat, Homogenization of elliptic equations with principal part not in divergence form and Hamiltonian with quadratic growth. Comm. Pure Appl. Math. 39 (1986) 769-805.

[14] A. Bensoussan, J.-L. Lions and G. Papanicolaou, Asymptotic Analysis for Periodic Structures. Corrected reprint of the 1978 original. AMS Chelsea Publishing, Providence, RI (2011).

[15] V.I. Bogachev and S.V. Shaposhnikov, Integrability and continuity of solutions to double divergence form equations. Ann. Mat. Pura Appl. 196 (2017) 1609-1635.

[16] V.I. Bogachev, N.V. Krylov and M. Röckner, On regularity of transition probabilities and invariant measures of singular diffusions under minimal conditions. Comm. Partial Differ. Equ. 26 (2001) 2037-2080.

[17] Y. Capdeboscq, Homogenization of a diffusion equation with drift. C. R. Acad. Sci. Paris Sér. I Math. 327 (1998) $807-812$.

[18] D. Cioranescu and P. Donato, An introduction to homogenization. In: Vol. 17 of Oxford Lecture Series in Mathematics and its Applications. The Clarendon Press, Oxford University Press, New York (1999).

[19] Y. Efendiev and T.Y. Hou, Multiscale finite element methods. Theory and applications. In: Vol. 4 of Surveys and Tutorials in the Applied Mathematical Sciences. Springer, New York (2009).

[20] A. Fannjiang and G. Papanicolaou, Convection enhanced diffusion for periodic flows. SIAM J. Appl. Math. 54 (1994) $333-408$.

[21] X. Feng, L. Hennings and M. Neilan, Finite element methods for second order linear elliptic partial differential equations in non-divergence form. Math. Comput. 86 (2017) 2025-2051.

[22] B.D. Froese and A.M. Oberman, Numerical averaging of non-divergence structure elliptic operators. Commun. Math. Sci. 7 (2009) 785-804.

[23] D. Gallistl, Variational formulation and numerical analysis of linear elliptic equations in nondivergence form with Cordes coefficients. SIAM J. Numer. Anal. 55 (2017) 737-757.

[24] D. Gallistl and E. Süli, Mixed finite element approximation of the Hamilton-Jacobi-Bellman equation with Cordes coefficients. SIAM J. Numer. Anal. 57 (2019) 592-614.

[25] D. Gilbarg and N.S. Trudinger, Elliptic partial differential equations of second order. Reprint of the 1998 edition. Classics in Mathematics. Springer-Verlag, Berlin (2001).

[26] P. Grisvard, Elliptic problems in nonsmooth domains. In: Vol. 69 of Classics in Applied Mathematics. Reprint of the 1985 original. Society for Industrial and Applied Mathematics (SIAM), Philadelphia, PA (2011).

[27] F. Hecht, New development in FreeFem++. J. Numer. Math. 20 (2012) 251-265.

[28] T. Hell and A. Ostermann, Compatibility conditions for Dirichlet and Neumann problems of Poisson's equation on a rectangle. J. Math. Anal. Appl. 420 (2014) 1005-1023.

[29] T. Hell, A. Ostermann and M. Sandbichler, Modification of dimension-splitting methods - overcoming the order reduction due to corner singularities. IMA J. Numer. Anal. 35 (2015) 1078-1091.

[30] P. Henning and M. Ohlberger, A note on homogenization of advection-diffusion problems with large expected drift. Z. Anal. Anwend. 30 (2011) 319-339.

[31] G. Iyer, T. Komorowski, A. Novikov and L. Ryzhik, From homogenization to averaging in cellular flows. Ann. Inst. Henri Poincaré Anal. Non Linéaire 31 (2014) 957-983.

[32] T. Kinoshita, Y. Watanabe, N. Yamamoto and M.T. Nakao, Some remarks on a priori estimates of highly regular solutions for the Poisson equation in polygonal domains. Jpn. J. Ind. Appl. Math. 33 (2016) 629-636.

[33] O. Lakkis and T. Pryer, A finite element method for second order nonvariational elliptic problems. SIAM J. Sci. Comput. 33 (2011) 786-801.

[34] A.H. Schatz, An observation concerning Ritz-Galerkin methods with indefinite bilinear forms. Math. Comput. 28 (1974) 959-962.

[35] I. Smears and E. Süli, Discontinuous Galerkin finite element approximation of nondivergence form elliptic equations with Cordès coefficients. SIAM J. Numer. Anal. 51 (2013) 2088-2106.

[36] I. Smears and E. Süli, Discontinuous Galerkin finite element approximation of Hamilton-Jacobi-Bellman equations with Cordes coefficients. SIAM J. Numer. Anal. 52 (2014) 993-1016.

[37] L. Tartar, The general theory of homogenization. A personalized introduction. In: Vol. 7 of Lecture Notes of the Unione Matematica Italiana. Springer-Verlag, Berlin; UMI, Bologna (2009).

[38] W. E B. Engquist, The heterogeneous multiscale methods. Commun. Math. Sci. 1 (2003) 87-132. 